This article was originally published in the Journal of Theoretical Probability, April 2004 .

The original publication is available at www.springerlink.com

DOI: $10.1023 /$ B: JOTP. $0000020701.20424 . d 8$

\title{
An Extension of the Contraction Principle
}

\author{
Jorge Garcia \\ Department of Mathematics \\ California State University Channel Islands \\ Thousand Oaks CA, 91360
}

\begin{abstract}
The concept of quasi-continuity and the new concept of almost compactness for a function are the basis for the extension of the contraction principle in large deviations presented here. Important equivalences for quasi-continuity are proved in the case of metric spaces. The relation between the exponential tightness of a sequence of stochastic processes and the exponential tightness of its transform (via an almost compact function) is studied here in metric spaces. Counterexamples are given to the non-metric case. Relations between almost compactness of a function and the goodness of a rate function are studied. Applications of the main theorem are given, including to an approximation of the stochastic integral.
\end{abstract}

Keywords large, deviation, contraction, principle, extension.

July 7,2003

\section{Contents}

1 Introduction

2 Observations On Other Extensions

3 Quasi-Continuity

4 A Contraction Principle $\quad 14$

4.1 Lower Bound . . . . . . . . . . . . . . . . . . . . 15

4.2 Upper Bound . . . . . . . . . . . . . . . . . . . . 18

5 Almost Compactness and Goodness $\quad 21$

6 Almost Compactness and Exponential Tightness 24 


\section{Introduction}

The contraction principle (see for example [2, Theorem 4.2.1]) says that if we have a sequence of random variables satisfying a large deviation principle, we can transform these random variables under a continuous function and construct a new sequence that will satisfy the large deviation principle. The contraction principle is the analog of the continuous mapping theorem in weak convergence. Originally stated by Varadhan [11, Theorem 3.3] it has been extended twice by Puhalskii [9, Theorem 2.2] [10, Theorem 2.1], Dembo and Zeitouni [2, Theorem 4.2.23], Eichelsbacher and Schmock [8, Theorem 1.12], Arcones [1, Theorem 2.1] and Deuschel and Stroock [3, Lemma 2.1.4]. We discuss these extensions below.

In Sections 3 and 4, we develop the concepts of "quasi-continuity" and "almost compactness", and we give the extension of the contraction principle, with the main result being the following.

Theorem 1.1 Assume $\Omega \stackrel{X_{n}}{\longrightarrow} \mathcal{X} \stackrel{G}{\longrightarrow} \mathcal{Y}, \mathcal{X}, \mathcal{Y}$ are metric spaces, and $\left\{X_{n}\right\}$ satisfies the large deviation principle with good rate function $I^{\sharp}$. Define $G^{x}=\left\{y \in \mathcal{Y} \mid\left(\exists x_{n} \rightarrow x\right) \quad G\left(x_{n}\right) \rightarrow y\right\}$. If for every $x$ with $I^{\sharp}(x)<\infty, G$ satisfies

- Every sequence converging to $x$ has a subsequence along which the function $G$ converges.

- For every $y \in G^{x}$ there is a sequence $\left\{x_{n}\right\}$ converging to $x$ such that $G\left(x_{n}\right) \rightarrow y, G$ is continuous at $x_{n}$ and $I^{\sharp}\left(x_{n}\right) \rightarrow I^{\sharp}(x)$.

Then $\left\{\left(X_{n}, G\left(X_{n}\right)\right)\right\}$ satisfies the large deviation principle with good rate function $I_{0}$ defined by:

$$
I_{0}(x, y)=\left\{\begin{array}{rll}
I^{\sharp}(x) & : & y \in G^{x}, \\
\infty & : & \text { otherwise }
\end{array} .\right.
$$


In particular, $\left\{G\left(X_{n}\right)\right\}$ satisfies the large deviation principle with rate function I given by:

$$
I(y)=\inf \left\{I_{0}(x, y): y \in G^{x}\right\}=\inf \left\{I^{\sharp}(x): y \in G^{x}\right\} .
$$

We have some applications of the main results from Sections 3 and 4

Corollary 1.2 Assume $\left\{X_{n}\right\} \in \mathbb{R}$ satisfies the large deviation principle with good rate function $I^{\sharp}$ and $G$ is a cadlag (caglad) function on the real line. If $I^{\sharp}$ is continuous at every $x$ where $G$ is discontinuous and $I^{\sharp}(x)<\infty$, then $\left\{\left(X_{n}, G\left(X_{n}\right)\right)\right\}$ satisfies the large deviation principle with rate function $I_{0}$ defined by 1.1. In particular, $\left\{G\left(X_{n}\right)\right\}$ satisfies the large deviation principle with rate function I defined by 1.2.

Corollary 1.3 If $\left\{X_{n}\right\} \in \mathbb{R}$ satisfies the large deviation principle upper bound and $G$ is a cadlag (caglad) function on the real line, then $\left\{\left(X_{n}, G\left(X_{n}\right)\right)\right\}$ satisfies the large deviation principle upper bound with a rate function defined by 1.1 .

Let $A^{\circ}$ and $\bar{A}$ denote the interior and the closure of a set $A$ respectively.

Corollary 1.4 Let $f_{i}: E \longrightarrow F$ be continuous functions between metric spaces and let $\left\{A_{i}\right\}$ be a measurable partition of $E$ such that for all $i, \overline{A_{i}^{\circ}}=\overline{A_{i}}$. If $X_{n} \in E$ satisfies the large deviation principle with good rate function $I^{\sharp}$ continuous on $\left\{x \mid I^{\sharp}(x)<\infty,(\exists i) x \in \partial A_{i}\right\}$ and $f=f_{i}$ on $A_{i}$, then $\left\{\left(X_{n}, f\left(X_{n}\right)\right)\right\}$ satisfies the large deviation principle with good rate function $I_{0}$ defined by 1.1. In particular, $\left\{f\left(X_{n}\right)\right\}$ satisfies the large deviation principle with a rate function defined by 1.2 .

In Section 3, we are primarily interested in functions that are not continuous. That is why the definitions of quasi- continuity and almost compactness play an important role in the desired extension. These concepts are important by themselves as can be appreciated 
in metric spaces under the equivalence presented in the first theorems of Sections 3 and 4. Cadlag and caglad functions are quasi-continuous and almost compact for example. In Section 4, before the above theorem is studied, we present two theorems as extensions of the contraction principle upper bound and lower bound respectively. The main theorem follows immediately from these results. In this same section we develop the concept of almost compactness to achieve a large deviation principle upper bound.

Section 5 briefly discusses the relation between the functions $G, I^{\sharp}$ and $I_{0}$ as defined in the previous section, but here no large deviation principle is assumed. Here we also study the relation between the "almost compactness" of the function $G$ and the goodness of the rate function $I^{\sharp}$. We find interesting equivalences among them, which allow us to say that the almost compact functions are the "right functions" to look at for purposes of an extension of the contraction principle. It is interesting to say that the extension presented here, is mainly a topological extension, not a "probabilistic extension" because the hypotheses have to do merely with the topological structure of the space; the basic assumption is that the function $G$ and the rate function $I^{\sharp}$ are "topologically related". This extension, in this sense, is different from many others.

Section 6 focuses on the relation between exponential tightness of a sequence of stochastic processes and the "almost compactness" of a function $G$. Statements of almost compactness in locally compact and metric spaces are studied, a diagram is provided to illustrate how almost compactness may be checked in different cases. This section includes two counterexamples to the following assertions that hold in metric spaces but not on general topological spaces

- $K$ compact and $G$ almost compact implies that the closure of $G(K)$ is compact

- a sequence $\left\{X_{n}\right\}$ of random variables being exponentially tight plus $G$ almost compact implies that $G\left(X_{n}\right)$ is exponentially tight. 
Finally Section 7 discusses an application of the main theorem to $\epsilon-$ approximations to the stochastic integral.

\section{Observations On Other Extensions}

Precisely, the contraction principle is as follows:

Theorem 2.1 Contraction Principle Let $\mathcal{X}, \mathcal{Y}$ be Hausdorff topological spaces and $f$ : $\mathcal{X} \longrightarrow \mathcal{Y}$ a continuous function. If a sequence of random variables $X_{n}$ satisfies the large deviation principle with good rate function $I$ on $\mathcal{X}$ and if we define

$$
I^{\prime}(y)=\inf \{I(x): f(x)=y\}
$$

then $f\left(X_{n}\right)$ satisfies the large deviation principle on $\mathcal{Y}$ with good rate function $I^{\prime}$.

Puhalskii [9, Theorem 2.2] extends the contraction principle to the case where the function is continuous at each point where the rate function is finite.

Dembo and Zeitouni [2, Theorem 4.2.23] have the following extension of the contraction principle:

Theorem 2.2 Let $\left\{X_{\epsilon}\right\}$ be a family of random variables that satisfies the large deviation principle with good rate function $I$ on a Hausdorff topological space $\mathcal{X}$, and for $m=1,2, .$. , let $f_{m}: \mathcal{X} \longrightarrow \mathcal{Y}$ be continuous functions, with $(\mathcal{Y}, d)$ a metric space. Assume there exists a measurable map $f: \mathcal{X} \longrightarrow \mathcal{Y}$ such that for every $\alpha<\infty$,

$$
\limsup _{m \rightarrow \infty} \sup _{\{x: I(x) \leq \alpha\}} d\left(f_{m}(x), f(x)\right)=0 .
$$

If $\left\{\hat{X}_{\epsilon}\right\}$ is a sequence of random variables such that $\left\{f_{m}\left(X_{\epsilon}\right)\right\}$ are exponentially good approximations of $\left\{\hat{X}_{\epsilon}\right\}$, then $\left\{\hat{X}_{\epsilon}\right\}$ satisfies the large deviation principle in $\mathcal{Y}$ with the good rate function $I^{\prime}(y)=\inf \{I(x): y=f(x)\}$. 
In their book, according to the remarks following this previous theorem, Dembo and Zeitouni claim that if for each $\epsilon>0, f$ restricted to the level set $\{x: I(x) \leq \alpha\}$ is continuous and if the rate function $I$ satisfies

$$
\lim _{m \rightarrow \infty} \inf _{x \in\{z: I(z)>m\}} I(x)=\infty,
$$

then their theorem implies that $f\left(X_{n}\right)$ satisfies the large deviation principle with some rate function, provided that $X_{n}$ does. The claim is, indeed, true. However these conditions on $f$ and $I$ imply that $f$ is continuous at each $x$ satisfying $I(x)<\infty$, and the conclusion follows by Puhalskii $[9$, Theorem 2.2].

Remark 2.3 If for every $\alpha>0, f$ restricted to the level set $\{x: I(x) \leq \alpha\}$ is continuous and the rate function I satisfies Condition 2.1, then $f$ is continuous at each $x$ satisfying $I(x)<\infty$.

Proof: Let $x$ satisfy $I(x) \leq \alpha<\infty$. Then it can not happen that there is a sequence $\left\{x_{n}\right\}$ converging to $x$ such that $I\left(x_{n}\right)$ converges to infinity. (Otherwise, for all $m, x$ would be in $\overline{\{z: I(z)>m\}}$ and then for all $m$

$$
\inf \{I(u): u \in \overline{\{z: I(z)>m\}}\} \leq I(x) \leq \alpha
$$

which is impossible.)

Therefore if $I(x) \leq \alpha<\infty$ and $x_{n}$ converges to $x$, then $\varlimsup_{n} I\left(x_{n}\right)<\infty$, and there exists $M$ such that all the values $x_{n}, n \geq M$ are inside of a level set. But restricted to this level set, $f$ is continuous. Hence $f\left(x_{n}\right)$ converges to $f(x)$.

Eischelsbacher \& Schmock [8, Theorem 1.12] extended the result from Dembo \& Zeitouni (Theorem 4.2.23) to completely regular topological spaces instead of metric spaces. In this 
article, the topology is changed to a "gauge" (open sets are generated by a family of balls with different pseudometrics), and the concept of exponentially good approximation is extended.

Puhalskii [10, Theorem 2.1] gives the following extension.

Theorem 2.4 If a sequence $\left\{X_{n}\right\}$ of random variables that takes values in a metric space $E$ satisfies the large deviation principle with good rate function $I$, and $h, h_{n}: E \longrightarrow E^{\prime}\left(E^{\prime}\right.$ a metric space) measurable functions satisfy:

- For any $\alpha>0, h$ restricted to $\{x: I(x) \leq \alpha\}$ is continuous

- For all $x$ with $I(x)<\infty$ and for all $x_{n} \longrightarrow x$, we have $h_{n}\left(x_{n}\right) \longrightarrow h(x)$,

then $\left\{h_{n}\left(X_{n}\right)\right\}$ satisfies the large deviation principle with rate function given by $I^{\prime}(y)=$ $\inf \{I(x): y=h(x)\}$

In this theorem, the second hypothesis implies the first one, but before proving this fact, consider the following extension due to Arcones [1, Theorem 2.1].

Theorem 2.5 If $X_{n}$ satisfies the large deviation principle with good rate function $I$ and $f$ is a measurable function between two metric spaces and $\left\{f_{n}\right\}$ is a sequence of measurable functions such that for all $x$ with $I(x)<\infty$ and for all $x_{n} \longrightarrow x$, we have $f_{n}\left(x_{n}\right) \longrightarrow f(x)$, then a large deviation principle holds for $f_{n}\left(X_{n}\right)$, and if it happens that for each $x$ with $I(x)<\infty, f$ is continuous at $x$, then the rate function for the sequence $\left\{f_{n}\left(X_{n}\right)\right\}$ is good.

The result of Arcones looks more general than the previous one from Puhalskii, but in fact, it is not.

Remark 2.6 If for all $x$ with $I(x)<\infty$ and for every sequence $\left\{x_{n}\right\}$ converging to $x$ we have $f_{n}\left(x_{n}\right) \longrightarrow f(x)$, then for all $\alpha>0, f$ restricted to $\{x: I(x) \leq \alpha\}$ is continuous. 
Proof: Note that if $I(z)<\infty$, then $f_{n}(z) \rightarrow f(z)$. Let $\alpha>0$ and $x, x_{k}$ such that $I(x), I\left(x_{k}\right) \leq \alpha$ and $x_{k} \rightarrow x$. We want to prove that $f\left(x_{k}\right) \rightarrow f(x)$. From the above observation: $f_{n}\left(x_{k}\right) \rightarrow f\left(x_{k}\right)$ as $n$ goes to $\infty$, therefore we can find $n_{k}$ with $d\left(f_{n_{k}}\left(x_{k}\right), f\left(x_{k}\right)\right)<1 / k$, with $n_{1}<n_{2}<\cdots<n_{k} \rightarrow \infty$

Let $z_{i}$ defined by $z_{i}=x_{1}$ for $1 \leq i \leq n_{1}$ and $z_{i}=x_{k}$ for $n_{k-1}<i \leq n_{k}$. Clearly $z_{i} \rightarrow x$ as $i \rightarrow \infty$, so $f_{i}\left(z_{i}\right) \rightarrow f(x)$ as $i \rightarrow \infty$. In particular $f_{n_{k}}\left(z_{n_{k}}\right) \rightarrow f(x)$, and hence

$$
\begin{aligned}
d\left(f\left(x_{k}\right), f(x)\right) & <d\left(f\left(x_{k}\right), f_{n_{k}}\left(z_{n_{k}}\right)\right)+d\left(f_{n_{k}}\left(z_{n_{k}}\right), f(x)\right) \\
& =d\left(f\left(x_{k}\right), f_{n_{k}}\left(x_{k}\right)\right)+d\left(f_{n_{k}}\left(z_{n_{k}}\right), f(x)\right) \longrightarrow 0 .
\end{aligned}
$$

Then $f$ restricted to $\{x: I(x) \leq \alpha\}$, is continuous

Deuschel and Stroock [3, Lemma 2.1.4] give the following extension.

Theorem 2.7 Let $\left\{X_{\epsilon}\right\}$ be a family of random variables that satisfies the large deviation principle with good rate function $I$ on a separable metric space $\mathcal{X}$, and for $m=1,2, .$. , let $f_{m}: \mathcal{X} \longrightarrow \mathcal{Y}$ be continuous functions, with $(\mathcal{Y}, d)$ another separable metric space. Assume there exists a measurable map $f: \mathcal{X} \longrightarrow \mathcal{Y}$ such that for every $\alpha \in[0, \infty)$,

$$
\lim \sup _{m \rightarrow \infty} \sup _{\{x: I(x) \leq \alpha\}} d\left(f_{m}(x), f(x)\right)=0 .
$$

Then $I^{\prime}(y)=\inf \{I(x): y=f(x)\}$ is a good rate function. Moreover, if, in addition

$$
\lim _{m \rightarrow \infty} \limsup _{\epsilon \rightarrow 0} \epsilon \log P\left[d\left(f\left(X_{\epsilon}\right), f_{m}\left(X_{\epsilon}\right)\right) \geq \delta\right]=-\infty
$$

for all $\delta>0$, then $\left\{f\left(X_{\epsilon}\right)\right\}$ satisfies the large deviation principle in $\mathcal{Y}$ with the good rate function $I$.

Equation 2.2 says that $\left\{f_{m}\left(X_{\epsilon}\right)\right\}$ are exponentially good approximations of $\left\{f\left(X_{\epsilon}\right)\right\}$, therefore this theorem can be obtained directly from Theorem 2.2. We note then that in Theorem 2.7 separability is not needed, since Theorem 2.2 refers to $\mathcal{X}$ as a Housdorff space. 
Deuschel and Stroock [3, Exercise 2.1.20], present five variations of Theorem 2.7, we will talk here about the first, where the space is modified, and the second, where the function is modified.

Assume that $\left\{X_{\epsilon}\right\}$ satisfies the large deviation principle with good rate function $I$ on a separable metric space $\mathcal{X}$. Let $(\mathcal{Y}, d)$ be another separable metric space.

Variation 2.8 Let $\left\{F_{L}: L \geq 0\right\}$ be a non-decreasing family of closed sets and $F_{\infty}=\bigcup_{L \geq 0} F_{L}$ such that for every $\epsilon>0, P\left[X_{\epsilon} \in F_{\infty}\right]=1$ and such that for every $L \geq 0$,

$$
\limsup _{\epsilon \rightarrow 0} \epsilon \log P\left[X_{\epsilon} \notin \overline{F_{L}}\right] \leq-L
$$

If $f: F_{\infty} \rightarrow \mathcal{Y}$ is a function whose restriction to each $F_{L}, L \geq 0$ is continuous, then $\left\{f\left(X_{\epsilon}\right)\right\}$ restricted to $F_{\infty}$, satisfies the large deviation principle, with the good rate function $I^{\prime}$ given by $I^{\prime}(y)=\inf \left\{I(x): x \in F_{\infty}, y=f(x)\right\}$.

Variation 2.8 can be obtained quickly by noticing that the set where the rate function $I$ is finite, is a subset of $F_{\infty}$, therefore using Lemma 4.1 .5 (b) in [2] we obtain that $\left\{X_{\epsilon}\right\}$ satisfies the large deviation principle on $F_{\infty}$ with rate function $\hat{I}$ such that $\hat{I}=I$ on $F_{\infty}$ and $\hat{I}=\infty$ on the complement of $F_{\infty}$. If we consider $f_{L}$ a continuous extension to $F_{\infty}$ of the restriction of $f$ to $F_{L}$, we can now apply Theorem 2.7 on the set $F_{\infty}$.

Variation 2.9 Let $\left\{f_{\epsilon}: \epsilon \geq 0\right\}$ be family of continuous maps from $\mathcal{X}$ into $\mathcal{Y}$. If

$$
\limsup _{\epsilon \rightarrow 0} \epsilon \log P\left[d\left(f_{\epsilon}\left(X_{\epsilon}\right), f_{0}\left(X_{\epsilon}\right)\right) \geq \delta\right]=-\infty
$$

for all $\delta>0$, then $\left\{f_{\epsilon}\left(X_{\epsilon}\right)\right\}_{\epsilon>0}$ satisfies the large deviation principle, with the good rate function $I^{\prime}$ given by $I^{\prime}(y)=\inf \{I(x): y=f(x)\}$.

Variation 2.9 can hold even without the continuity of the functions $f_{\epsilon}$ for $\epsilon>0$, as stated in Corollary 4.2.21 of [2], mainly because equation 2.4 allows us to conclude that $\left\{f_{\epsilon}\left(X_{\epsilon}\right)\right\}$ 
are exponentially equivalent to $\left\{f_{0}\left(X_{\epsilon}\right)\right\}$, which, by the contraction principle, satisfies the large deviation principle, and therefore by a standard result (see [2, Theorem 4.2.13]) we conclude that the same large deviation principle holds for $\left\{f_{\epsilon}\left(X_{\epsilon}\right)\right\}$.

The previous extensions consider a sequence of measurable functions and conclude a large deviation principle for $f_{n}\left(X_{n}\right)$, except the one from Puhalskii [9, Theorem 2.2] and the one from Deuschel and Stroock [3, Theorem 2.1.4].

The extension presented here is different from the previous ones. Here we conclude a large deviation principle for $f\left(X_{n}\right)$ for a discontinuous function $f$.

The examples described in Section 7 and Corollaries 1.2, 1.3 and 1.4, are examples where

Puhalskii's extension [9, Theorem 2.2] or Deuschel and Stroock's extension [3, Theorem 2.1.4] does not apply.

\section{Quasi-Continuity}

Let $\mathcal{X}, \mathcal{Y}$ be topological spaces and let $G$ be a function from $\mathcal{X}$ to $\mathcal{Y}$. We will denote a net in $\mathcal{X}$ by $\left\{x_{\alpha}\right\}$ and a sequence by $\left\{x_{n}\right\}$. Define

$$
G^{x}=\left\{y \in \mathcal{Y} \mid\left(\exists x_{\alpha} \rightarrow x\right) \quad G\left(x_{\alpha}\right) \rightarrow y\right\}
$$

Definition 3.1 We say that $G$ is quasi-continuous at $x \in \mathcal{X}$ if for every $y \in G^{x}$ and every pair $(\mathcal{U}, \mathcal{W})$ of neighborhoods of $x$ and $y$ respectively, there is a non-empty open subset $\mathcal{U}_{0}$ of $\mathcal{U}$ such that $G\left(\mathcal{U}_{0}\right) \subseteq \mathcal{W}$

We say that the function $G$ is quasi-continuous if it is quasi-continuous at every point of its domain.

Quasi-continuity is an old concept. In 1899, Baire stated that first Volterra observed the fact that every separately continuous function $f: \mathbb{R} \times \mathbb{R} \longrightarrow \mathbb{R}$ is quasi-continuous. It 
was Kempisty [7, pp.185] who called this property of separately continuous functions quasicontinuity. This concept has been studied by Gangster [4]. In particular he relates different notions of continuity. This concept is also known as semicontinuity.

Note that any continuous function is quasi-continuous. We give useful ways of checking that a function is quasi-continuous.

We will use the notation $V^{\text {open }}$ to denote that the set $V$ is open.

Theorem 3.2 The following are equivalent.

1. $G$ is quasi-continuous

2. For each $x \in \mathcal{X}, \mathcal{U}$ open containing $x$ and $\mathcal{W}$ open containing $G(x)$, there is a nonempty open subset $\mathcal{U}_{0}$ of $\mathcal{U}$ such that $G\left(\mathcal{U}_{0}\right) \subseteq \mathcal{W}$

If the spaces are metric and complete, then the previous two conditions are equivalent to the following three:

3. For each $x \in \mathcal{X}$, there is a sequence $\left\{x_{n}\right\}$ such that $x_{n} \rightarrow x, G\left(x_{n}\right) \rightarrow G(x)$ and such that for all $n, G$ is continuous at $x_{n}$

4. For each $x \in \mathcal{X}$, there is a sequence $\left\{x_{n}\right\}$ such that $x_{n} \rightarrow x, G\left(x_{n}\right) \rightarrow G(x)$ and such that for all $n, G$ is quasi-continuous at $x_{n}$

5. For each $x \in \mathcal{X}, y \in G^{x}$ there is a sequence $\left\{x_{n}\right\}$ such that $x_{n} \rightarrow x, G\left(x_{n}\right) \rightarrow y$ and such that for all $n, G$ is continuous at $x_{n}$

\section{Proof:}

$[\mathbf{1} \Rightarrow \mathbf{2}]$ Let $x \in \mathcal{X}$. Then $y=G(x)$ can be written as the limit of $G\left(x_{\gamma}\right)$ where $\left\{x_{\gamma}\right\}$ is a net converging to $x$ (namely $x_{\gamma} \equiv x$ ). Let $\mathcal{U}^{\text {open }} \ni x, \mathcal{W}^{\text {open }} \ni y=G(x)$. By hypothesis there is a non-empty open subset $\mathcal{U}_{0}$ of $\mathcal{U}$ such that $G\left(\mathcal{U}_{0}\right) \subseteq \mathcal{W}$. 
$[\mathbf{2} \Rightarrow \mathbf{1}]$ Let $x \in \mathcal{X}, y \in \mathcal{Y}$ such that there is a net $\left\{x_{\gamma}\right\}$ converging to $x$ with $G\left(x_{\gamma}\right) \rightarrow y$ and let $\mathcal{U}^{\text {open }} \ni x, \mathcal{W}^{\text {open }} \ni y$. There is $\gamma_{1}$ such that if $\gamma \geq \gamma_{1}$, then $x_{\gamma} \in \mathcal{U}$ and there is $\gamma_{2}$ such that if $\gamma \geq \gamma_{2}$, then $G\left(x_{\gamma}\right) \in \mathcal{W}$. Take $\gamma_{3} \geq \gamma_{1}, \gamma_{2}$, then $x_{\gamma_{3}} \in \mathcal{U}, G\left(x_{\gamma_{3}}\right) \in \mathcal{W}$. From hypothesis, there is a non-empty open subset $\mathcal{U}_{0}$ of $\mathcal{U}$ such that $G\left(\mathcal{U}_{0}\right) \subseteq \mathcal{W}$.

$[\mathbf{2} \Rightarrow \mathbf{3}]$ (In the case of a metric space). Let $\mathcal{U}_{0}$ be any non-empty open subset of $\mathcal{X}, r_{1} \in \mathcal{U}_{0}$ and $\mathcal{W}_{0}$ any open set containing $G\left(r_{1}\right)$. Take $n_{1}$ a positive integer such that $B_{1 / n_{1}}\left(r_{1}\right) \subseteq \mathcal{U}_{0}$ and $B_{1 / n_{1}}\left(G\left(r_{1}\right)\right) \subseteq \mathcal{W}_{0}$

Applying the hypothesis to $r_{1}, B_{1 / 2 n_{1}}\left(r_{1}\right), B_{1 / 2 n_{1}}\left(G\left(r_{1}\right)\right)$ we have that there is an open nonempty subset $\mathcal{U}_{1}$ of $B_{1 / 2 n_{1}}\left(r_{1}\right)$ whose image is inside $B_{1 / 2 n_{1}}\left(G\left(r_{1}\right)\right)$. Take $r_{2} \in \mathcal{U}_{1}, n_{2}>n_{1}$ such that $B_{1 / n_{2}}\left(r_{2}\right) \subseteq \mathcal{U}_{1}, B_{1 / n_{2}}\left(G\left(r_{2}\right)\right) \subseteq B_{1 / 2 n_{1}}\left(G\left(r_{1}\right)\right)$.

Applying the hypothesis to $r_{2}, B_{1 / 2 n_{2}}\left(r_{2}\right), B_{1 / 2 n_{2}}\left(G\left(r_{2}\right)\right)$, we have that there is an open nonempty subset $\mathcal{U}_{2}$ of $B_{1 / 2 n_{2}}\left(r_{2}\right)$ whose image is inside $B_{1 / 2 n_{2}}\left(G\left(r_{2}\right)\right)$. Take $r_{3} \in \mathcal{U}_{2}, n_{3}>n_{2}$ such that $B_{1 / n_{3}}\left(r_{3}\right) \subseteq \mathcal{U}_{2}, B_{1 / n_{3}}\left(G\left(r_{3}\right)\right) \subseteq B_{1 / 2 n_{2}}\left(G\left(r_{2}\right)\right)$.

If we continue in this way, we can find sequences: $\left\{r_{k}\right\},\left\{n_{k}\right\}$, such that:

$$
\begin{aligned}
B_{1 / n_{1}}\left(r_{1}\right) & \supseteq B_{1 / 2 n_{1}}\left(r_{1}\right) \supseteq B_{1 / n_{2}}\left(r_{2}\right) \supseteq B_{1 / 2 n_{2}}\left(r_{2}\right) \supseteq B_{1 / n_{3}}\left(r_{3}\right) \cdots \\
B_{1 / n_{1}}\left(G\left(r_{1}\right)\right) & \supseteq B_{1 / 2 n_{1}}\left(G\left(r_{1}\right)\right) \supseteq B_{1 / n_{2}}\left(G\left(r_{2}\right)\right) \supseteq B_{1 / 2 n_{2}}\left(G\left(r_{2}\right)\right) \cdots
\end{aligned}
$$

Therefore we obtain that:

$$
\begin{aligned}
B_{1 / n_{1}}\left(r_{1}\right) & \supseteq \overline{B_{1 / 2 n_{1}}\left(r_{1}\right)} \supseteq B_{1 / n_{2}}\left(r_{2}\right) \supseteq \overline{B_{1 / 2 n_{2}}\left(r_{2}\right)} \supseteq B_{1 / n_{3}}\left(r_{3}\right) \cdots \\
B_{1 / n_{1}}\left(G\left(r_{1}\right)\right) & \supseteq \overline{B_{1 / 2 n_{1}}\left(G\left(r_{1}\right)\right)} \supseteq B_{1 / n_{2}}\left(G\left(r_{2}\right)\right) \supseteq \overline{B_{1 / 2 n_{2}}\left(G\left(r_{2}\right)\right)} \cdots
\end{aligned}
$$

Let $r \in \bigcap_{k} \overline{B_{1 / 2 n_{k}}\left(r_{k}\right)}$ and $s \in \bigcap_{k} \overline{B_{1 / 2 n_{k}}\left(G\left(r_{k}\right)\right)}$.

Since for any $k, G\left(B_{1 / n_{k+1}}\left(r_{k+1}\right)\right) \subseteq G\left(\mathcal{U}_{k}\right) \subseteq B_{1 / 2 n_{k}}\left(G\left(r_{k}\right)\right)$, we obtain

$$
\begin{aligned}
G(r) & \in G\left(\bigcap_{k} \overline{B_{1 / 2 n_{k+1}}\left(r_{k+1}\right)}\right) \subseteq \bigcap_{k} G\left(\overline{B_{1 / 2 n_{k+1}}\left(r_{k+1}\right)}\right) \\
& \subseteq \bigcap_{k} B_{1 / 2 n_{k}}\left(G\left(r_{k}\right)\right) \subseteq \bigcap_{k} \overline{B_{1 / 2 n_{k}}\left(G\left(r_{k}\right)\right)}=\{s\} .
\end{aligned}
$$


Therefore $G(r)=s$. It is clear that $r \in \mathcal{U}_{0}$ and that $G(r)=s \in \mathcal{W}_{0}$

Claim $G$ is continuous at $r$.

Proof (claim) Let $\mathcal{W}^{\text {open }} \ni s$, then there is $k$ with

$$
\mathcal{W} \supseteq B_{1 / n_{k}}\left(G\left(r_{k}\right)\right) \supseteq \overline{B_{1 / 2 n_{k}}\left(G\left(r_{k}\right)\right)}
$$

Since $B_{1 / n_{k+1}}\left(r_{k+1}\right) \subseteq \mathcal{U}_{k}$, then $G\left(B_{1 / n_{k+1}}\left(r_{k+1}\right)\right) \subseteq G\left(\mathcal{U}_{k}\right) \subseteq B_{1 / 2 n_{k}}\left(G\left(r_{k}\right)\right) \subseteq \mathcal{W}$.

But $r \in \overline{B_{1 / 2 n_{k+1}}\left(r_{k+1}\right)} \subseteq B_{1 / n_{k+1}}\left(r_{k+1}\right)$, therefore $G$ is continuous at $r$.

We just proved that for any triple $\left(r_{1}, \mathcal{U}_{0}, \mathcal{W}_{0}\right)$ such that $\mathcal{U}_{0}, \mathcal{W}_{0}$ are open, $r_{1} \in U_{0}$ and $G\left(r_{1}\right) \in \mathcal{W}_{0}$ we can find $r \in \mathcal{U}_{0}$ such that $G$ is continuous at $r$ and $G(r) \in \mathcal{W}_{0}$.

If $x \in \mathcal{X}$, there is a sequence $\left\{x_{n}\right\}$ such that for each $n$

$$
x_{n} \in B_{1 / n}(x), G\left(x_{n}\right) \in B_{1 / n}(G(x)), G \text { is continuous at } x_{n}
$$

Therefore there is a sequence $\left\{x_{n}\right\}$ such that $x_{n} \rightarrow x, G\left(x_{n}\right) \rightarrow G(x)$ and for all $n, G$ is continuous at $x_{n}$.

$$
[3 \Rightarrow 4] \text { Trivial. }
$$

$[\mathbf{4} \Rightarrow \mathbf{2}]$ Let $\mathcal{U}^{\text {open }} \ni x, \mathcal{W}^{\text {open }} \ni G(x)$. Let $\left\{x_{n}\right\}$ be a sequence with $\left\{x_{n}\right\} \rightarrow x,\left\{G\left(x_{n}\right)\right\} \rightarrow$ $G(x)$ and $G$ continuous at $x_{n}$. Take a positive integer $N$ such that $x_{N} \in \mathcal{U}, G\left(x_{N}\right) \in \mathcal{W}$. Since $G$ is quasi-continuous at $x_{N}$, there is an open non-empty subset $\mathcal{U}_{0}$ of $\mathcal{U}$ such that $G\left(\mathcal{U}_{0}\right) \subseteq \mathcal{W}$, and 2 follows.

$[\mathbf{3} \Rightarrow 5]$ Note that if $\mathcal{X}$ and $\mathcal{Y}$ are metric, we can replace "nets" by "sequences" in the definition of $G^{x}$. Let $x \in \mathcal{X}, y \in \mathcal{Y}, y \in G^{x}$. We can find $\left\{z_{n}\right\}$ with $z_{n} \rightarrow x, G\left(z_{n}\right) \rightarrow y$. By hypothesis, there is a sequence $\left\{z_{n}^{k}\right\}_{k}$ with $z_{n}^{k} \rightarrow z_{n},\left\{G\left(z_{n}^{k}\right)\right\} \rightarrow G\left(z_{n}\right)$ as $k \rightarrow \infty$ and for all $k, G$ is continuous at $z_{n}^{k}$. For each $n$ there is $k_{n}$ such that $z_{n}^{k_{n}} \in B_{1 / n}\left(z_{n}\right)$ and $G\left(z_{n}^{k_{n}}\right) \in B_{1 / n}\left(G\left(z_{n}\right)\right)$. If we let $x_{n}=z_{n}^{k_{n}}$ we have that: $x_{n} \rightarrow x, G\left(x_{n}\right) \rightarrow y$ and for all $n, G$ is continuous at $x_{n}$. 
$[\mathbf{5} \Rightarrow \mathbf{3}]$ Here we just apply the hypothesis to the point $y=G(x) \in G^{x}$ and the conclusion is exactly what we want to prove.

Observation The following conditions imply that $G$ is quasi-continuous:

1. For each $x \in \mathcal{X}$, there is a net $\left\{x_{\alpha}\right\}$ such that $x_{\alpha} \rightarrow x, G\left(x_{\alpha}\right) \rightarrow G(x)$ and such that for all $\alpha, G$ is continuous at $x_{\alpha}$

2. For each $x \in \mathcal{X}$, there is a net $\left\{x_{\alpha}\right\}$ such that $x_{\alpha} \rightarrow x, G\left(x_{\alpha}\right) \rightarrow G(x)$ and such that for all $\alpha, G$ is quasi-continuous at $x_{\alpha}$.

The proof is the same as the one: $\mathbf{4} \Rightarrow \mathbf{2}$

Some examples of quasi-continuous functions on $\mathbb{R}$ are all the cadlag and caglad functions, $G(x)=\sin \left(\frac{1}{x}\right), G(x)=\frac{1}{x} \sin \left(\frac{1}{x}\right)$ as long as we define $G(0) \in[-1,1]$ in the first case and $G(0) \in \mathbb{R}$ in the second case. The indicator of the rationals and the function $G(x)=1$ for $x<0, G(0)=2, G(x)=3$ for $x>0$ are not quasi-continuous function.

If $F$ is a continuous function and $G$ is quasi-continuous, then $F \circ G$ is quasi-continuous but $G \circ F$ is not necessarily quasi-continuous.

\section{A Contraction Principle}

In this section, we will assume the following situation: $\Omega \stackrel{X_{n}}{\longrightarrow} \mathcal{X} \stackrel{G}{\longrightarrow} \mathcal{Y}$ where $\mathcal{X}, \mathcal{Y}$ are topological Hausdorff spaces, $\left\{X_{n}\right\}$ satisfies the large deviation principle with rate function $I^{\sharp}$ and $G$ is a measurable function. For $y \in \mathcal{Y}$, let $\left\{B_{\beta}^{y}\right\}_{\beta \in \mathcal{B}_{y}}$ be a basis of open sets in $\mathcal{Y}$ containing $y$. Define $L_{n}^{x}=\left(-\infty, I^{\sharp}(x)+\frac{1}{n}\right]$, and

$$
I_{0}(x, y)=\left\{\begin{array}{rll}
I^{\sharp}(x) & : & y \in G^{x}, \\
\infty & : & \text { otherwise }
\end{array} .\right.
$$




\subsection{Lower Bound}

For a function $I$ taking non-negative values, and a subset $A$ of its domain, we denote by $I(A)$ the infimum of the values of $I$ on the subset $A$.

The following lemma will be helpful:

Lemma 4.1 Let $\left\{X_{n}\right\}$ be a sequence of random variables taking values on a product space $\mathcal{X} \times \mathcal{Y}$. If $\left\{X_{n}\right\}$ satisfies the large deviation principle lower bound on "open boxes", then $\left\{X_{n}\right\}$ satisfies the large deviation principle lower bound.

The previous lemma is standard and its proof can be found in [2, Theorem 4.2.1].

Lemma $4.2 I_{0}$ is lower semicontinuous.

Proof: Let $\left(x_{\alpha}, y_{\alpha}\right) \longrightarrow(x, y)$. If $y \in G^{x}$, then $I_{0}(x, y)=I^{\sharp}(x) \leq \liminf I^{\sharp}\left(x_{\alpha}\right) \leq$ $\liminf I_{0}\left(x_{\alpha}, y_{\alpha}\right)$. If $y \notin G^{x}$, then there are open sets $U, W$ containing $x$ and $y$ respectively such that $U \cap G^{-1}(W)=\emptyset$. Consider $\alpha_{0}$ such that if $\alpha \geq \alpha_{0}$, then $x_{\alpha} \in U, y_{\alpha} \in W$. Take $\alpha \geq \alpha_{0}$. If $y_{\alpha} \in G^{x_{\alpha}}$, then there is a net converging to $x_{\alpha}$ whose image under $G$ converges to $y_{\alpha}$. Therefore, $U \cap G^{-1}(W)$ would not be empty. Hence $y_{\alpha} \notin G^{x_{\alpha}}$, which implies that $I_{0}\left(x_{\alpha}, y_{\alpha}\right)=\infty$. Therefore, $I_{0}(x, y) \leq \liminf I_{0}\left(x_{\alpha}, y_{\alpha}\right)$.

Theorem 4.3 Suppose that for each $x$ such that $I^{\sharp}(x)<\infty$ and each $y \in G^{x}$

$$
x \in \bigcap_{n, \beta} \overline{I^{\sharp-1}\left(L_{n}^{x}\right) \cap\left(G^{-1}\left(B_{\beta}^{y}\right)\right)^{\circ}} .
$$

Then a large deviation principle lower bound holds for $\left\{\left(X_{n}, G\left(X_{n}\right)\right)\right\}$ with rate function $I_{0}$.

Proof: By the previous lemma, it is enough to prove the large deviation principle lower bound for $O=O_{1} \times O_{2}$, with $O_{1}, O_{2}$ open. Applying our hypothesis we obtain:

$$
\begin{aligned}
\underline{\lim } \frac{1}{n} \log P\left[\left(X_{n}, G\left(X_{n}\right)\right) \in O\right] & =\underline{\lim } \frac{1}{n} \log P\left[X_{n} \in O_{1} \bigcap G^{-1}\left(O_{2}\right)\right] \\
& \geq \underline{\lim } \frac{1}{n} \log P\left[X_{n} \in\left(O_{1} \cap G^{-1}\left(O_{2}\right)\right)^{\circ}\right] \\
& \geq-I^{\sharp}\left(\left(O_{1} \cap G^{-1}\left(O_{2}\right)\right)^{\circ}\right) .
\end{aligned}
$$


To finish, it would be enough to prove that $I_{0}(O) \geq I^{\sharp}\left(\left(O_{1} \cap G^{-1}\left(O_{2}\right)\right)^{\circ}\right)$. Equivalently: If $(x, y) \in O_{1} \times O_{2}$, we would like to prove that:

$$
I_{0}(x, y) \geq I^{\sharp}\left(\left(O_{1} \cap G^{-1}\left(O_{2}\right)\right)^{\circ}\right) .
$$

It is enough to prove (4.2) for $y \in G^{x}$. If $I^{\sharp}(x)=\infty$, then $I_{0}(x, y)=\infty$ so (4.2) holds. Then we can assume that $I^{\sharp}(x)<\infty$ and that $y \in G^{x}$.

Take $\beta \in \mathcal{B}_{y}$ with $B_{\beta}^{y} \subseteq O_{2}$ and take $n$ any natural number. Since $x \in O_{1}$ and, by hypothesis $x \in \overline{I^{\sharp-1}\left(L_{n}^{x}\right) \cap\left(G^{-1}\left(B_{\beta}^{y}\right)\right)^{\circ}}$, we can find a point $x_{n} \in O_{1}$ such that $x_{n} \in I^{\sharp}-1\left(L_{n}^{x}\right) \cap$ $\left(G^{-1}\left(B_{\beta}^{y}\right)\right)^{\circ}$. Therefore, $I^{\sharp}\left(x_{n}\right) \leq I^{\sharp}(x)+\frac{1}{n}$, and there is a non-empty open set $U_{n}$ containing $x_{n}$ with $G\left(U_{n}\right) \subseteq B_{\beta}^{y} \subseteq O_{2}$. Then $x_{n} \in O_{1}^{\circ} \cap\left(G^{-1}\left(O_{2}\right)\right)^{\circ}=\left(O_{1} \cap G^{-1}\left(O_{2}\right)\right)^{\circ}$, and therefore

$$
I^{\sharp}\left(\left(O_{1} \cap G^{-1}\left(O_{2}\right)\right)^{\circ}\right) \leq I^{\sharp}\left(x_{n}\right) \leq I^{\sharp}(x)+\frac{1}{n} .
$$

Taking $n \rightarrow \infty$, we obtain (4.2).

In the following theorem, given a function $f$ and a rate function $I, C_{f}$ represents the set of points where $f$ is continuous, $Q_{f}$ represents the set of points where $f$ is quasi-continuous, and $F_{I}$ represents the set of points where $I$ is finite.

Theorem 4.4 Any of the following conditions implies (4.1)

1. $G$ is quasi-continuous at $x$ and for every open set $\mathcal{U}$ whose closure contains $x$, there is a sequence $\left\{x_{n}\right\}$ inside $\mathcal{U}$ such that $I^{\sharp}\left(x_{n}\right) \rightarrow I^{\sharp}(x)$

2. $G$ is continuous at $x$

3. $G$ is quasi-continuous at $x$ and $I^{\sharp}(x)$ is continuous at $x$

4. For each $y \in G^{x}$, there is a sequence $\left\{x_{n}\right\}$ such that $x_{n} \rightarrow x, G\left(x_{n}\right) \rightarrow y, I^{\sharp}\left(x_{n}\right) \rightarrow$ $I^{\sharp}(x)$ and such that for all $n, G$ is continuous at $x_{n}$ 
5. For each $y \in G^{x}$, there is a net $\left\{x_{\alpha}\right\}$ such that $x_{\alpha} \rightarrow x, G\left(x_{\alpha}\right) \rightarrow G(x), I^{\sharp}\left(x_{\alpha}\right) \rightarrow I^{\sharp}(x)$ and such that for all $\alpha, G$ is continuous at $x_{\alpha}$.

Proof: Let $x, y$ with $I^{\sharp}(x)<\infty$ and $y \in G^{x}$

$[\mathbf{2} \Rightarrow(\mathbf{4 . 1})]$ Since $G$ is continuous and $I^{\sharp}(x)<\infty$ we really have: $y=G(x)$. Of course, if $n>0$, then $x \in I^{\sharp}-1\left(L_{n}^{x}\right) \cap\left(G^{-1}\left(B_{\beta}^{y}\right)\right)^{\circ}$.

$[3 \Rightarrow$ (4.1) $]$ Let $n \geq 1, O$ open, $O \ni x$ and $\beta \in \mathcal{B}_{y}$. Using the quasi-continuity of $G$ we can find a net $\left\{x_{\alpha}\right\} \subseteq O$ and a collection of open sets $\left\{U_{\alpha}\right\}$ such that: $x_{\alpha} \rightarrow x, x_{\alpha} \in$ $U_{\alpha}, G\left(U_{\alpha}\right) \subseteq B_{\beta}^{y}$ and $U_{\alpha} \subseteq O$

Using the continuity of $I^{\sharp}$ we can find $\alpha_{0}$ with $I^{\sharp}\left(x_{\alpha_{0}}\right) \leq I^{\sharp}(x)+\frac{1}{n}$, we also know that $x_{\alpha_{0}} \in U_{\alpha_{0}} \subseteq O$ and $G\left(U_{\alpha_{0}}\right) \subseteq B_{\beta}^{y}$.

Then $x_{\alpha_{0}} \in O \cap I^{\sharp}-1\left(L_{n}^{x}\right) \cap\left(G^{-1}\left(B_{\beta}^{y}\right)\right)^{\circ}$, hence $x \in \overline{I^{\sharp-1}\left(L_{n}^{x}\right) \cap\left(G^{-1}\left(B_{\beta}^{y}\right)\right)^{\circ}}$.

$[\mathbf{5} \Rightarrow(\mathbf{4 . 1})]$ Let $n \geq 1, O$ open, $O \ni x$ and $\beta \in \mathcal{B}_{y}$. By hypothesis, there is $\left\{x_{\alpha}\right\}$ a net such that $x_{\alpha} \rightarrow x, \quad G\left(x_{\alpha}\right) \rightarrow y, \quad G$ continuous at $x_{\alpha}$ and $I^{\sharp}\left(x_{\alpha}\right) \rightarrow I^{\sharp}(x)$.

Take $\alpha_{0}$ with $x_{\alpha_{0}} \in O, I^{\sharp}\left(x_{\alpha_{0}}\right) \leq I^{\sharp}(x)+\frac{1}{n}, G\left(x_{\alpha_{0}}\right) \in B_{\beta}^{y}$. Since $\mathrm{G}$ is continuous at $x_{\alpha_{0}}$, there is $U_{\alpha_{0}}$ open set containing $x_{\alpha_{0}}$ with $G\left(U_{\alpha_{0}}\right) \subseteq B_{\beta}^{y}$.

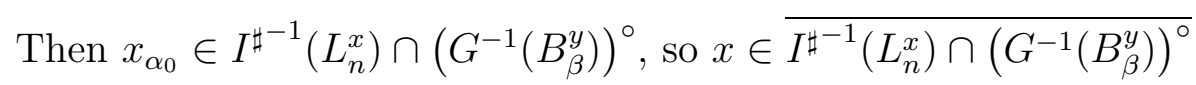

$[\mathbf{4} \Rightarrow \mathbf{( 4 . 1 )}]$ Any sequence is a net, so the result follows from: $5 \Rightarrow(\mathbf{4 . 1})$

$\left[\mathbf{1} \Rightarrow\right.$ (4.1)] Let $n \geq 1, O$ open, $O \ni x$ and $\beta \in \mathcal{B}_{y}$. We need to find a point $\hat{x}$ such that : a) $\hat{x} \in O$, b) $I^{\sharp}(\hat{x}) \leq I^{\sharp}(x)+\frac{1}{n}$ and $\left.\boldsymbol{c}\right) \hat{x} \in U \subseteq G^{-1}\left(B_{\beta}^{y}\right)$ for some open set $U$.

Apply the fact that $G$ is quasi-continuous to the sets $O, B_{\beta}^{y}$. Let $U$ be the maximal open subset of $O$ such that $G(U) \subseteq B_{\beta}^{y}$. Then $\bar{U} \ni x$ and $G(U) \subseteq B_{\beta}^{y}$. 
Let $\left\{x_{m}\right\} \subseteq U$ with $I^{\sharp}\left(x_{m}\right) \rightarrow I^{\sharp}(x)$. Take $m$ with $I^{\sharp}\left(x_{m}\right) \leq I^{\sharp}(x)+\frac{1}{n}$. Now $\hat{x}=x_{m}$ satisfies the above three conditions.

Corollary 4.5 If $F_{I^{\sharp}} \subseteq C_{G} \bigcup\left(C_{I^{\sharp}} \bigcap Q_{G}\right)$, then a large deviation principle lower bound holds for $\left\{\left(X_{n}, G\left(X_{n}\right)\right)\right\}$ with rate function $I_{0}$.

Proof: This proof follows from $2 \Rightarrow(4.1)$ and $3 \Rightarrow(4.1)$ in the previous theorem.

\subsection{Upper Bound}

Now we give an extension of the contraction principle upper bound:

Definition 4.6 We say that $G$ is an almost compact function at $x$ if for every net $\left\{x_{\alpha}\right\}$ converging to $x$, there is a subnet along which $G$ converges to a point $y \in \mathcal{Y}$. We say that $G$ is almost compact if it is almost compact at every $x \in \mathcal{X}$. Define $K_{G}$ to be the set of points where $G$ is almost compact.

Theorem 4.7 Let $G$ be a measurable function with respect to the Borel sigma-algebra in $\mathcal{X}$. Any of the following conditions implies that a large deviation principle upper bound holds for the couple $\left\{\left(X_{n}, G\left(X_{n}\right)\right)\right\}$ with rate function $I_{0}$.

1. For every $x \in F_{I^{\sharp}}$ and every net $\left\{x_{\gamma}\right\}$ converging to $x$ either there is a subnet $\left\{x_{\delta}\right\}$ along which $G$ converges to some point $y \in \mathcal{Y}$ or there is a subnet $\left\{x_{\delta}\right\}$ along which $I^{\sharp}(x)=\lim _{\delta} I^{\sharp}\left(x_{\delta}\right)$

2. (If the space $\mathcal{X}$ is metric) For every $x \in F_{I^{\sharp}}$ and every sequence $\left\{x_{n}\right\}$ converging to $x$ either there is a subsequence $\left\{x_{n_{k}}\right\}$ along which $G$ converges to some point $y \in \mathcal{Y}$ or there is a subsequence $\left\{x_{n_{k}}\right\}$ along which $I^{\sharp}(x)=\lim _{k} I^{\sharp}\left(x_{n_{k}}\right)$. 
Proof: Let $F$ be a closed set in the product space, and define $H_{F}=\{x \in \mathcal{X}:(x, G(x)) \in F\}$. Then

$$
\begin{aligned}
\varlimsup \frac{1}{n} \log P\left[\left(X_{n}, G\left(X_{n}\right)\right) \in F\right] & =\varlimsup \\
& \leq \varlimsup \frac{1}{n} \log P\left[X_{n} \in H_{F}\right] \\
& \leq-I^{\sharp}\left(\overline{H_{F}}\right) .
\end{aligned}
$$

To finish the proof of this theorem it would be enough to prove that each of the two conditions in the theorem implies $I_{0}(F) \leq I^{\sharp}\left(\overline{H_{F}}\right)$, or, equivalently, that for every $x \in \overline{H_{F}}$

$$
I_{0}(F) \leq I^{\sharp}(x)
$$

(If $\overline{H_{F}}=\emptyset$, then $I_{0}(F) \leq \infty=I^{\sharp}\left(\overline{H_{F}}\right)$, hence, we can assume that $\overline{H_{F}} \neq \emptyset$.) Let then $x$ be in $\overline{H_{F}}$ and $\left\{x_{\alpha}\right\} \subseteq H_{F}$ with $x=\lim x_{\alpha}$. We can assume that $I^{\sharp}(x)$ is finite (otherwise (4.3) would be trivial).

$[\mathbf{1} \Rightarrow(\mathbf{4 . 3})]$ Consider the net $x_{\alpha}$ that converges to $x$. Assume first that there is a subnet $\left\{x_{\beta}\right\}$ of $\left\{x_{\alpha}\right\}$ such that $\left\{G\left(x_{\beta}\right)\right\}$ converges. Let $y=\lim G\left(x_{\beta}\right)$. Then $y \in G^{x}$. Since $F \ni\left(x_{\beta}, G\left(x_{\beta}\right)\right)$ and $\left(x_{\beta}, G\left(x_{\beta}\right)\right) \rightarrow(x, y)$, we obtain that $(x, y) \in F$, therefore

$$
I_{0}(F) \leq I_{0}(x, y)=I^{\sharp}(x) .
$$

Finally assume that there is a subnet $x_{\delta}$ along which $I^{\sharp}(x)=\lim _{\delta} I^{\sharp}\left(x_{\delta}\right)$. Then $I_{0}(F) \leq$ $I_{0}\left(x_{\delta}, G\left(x_{\delta}\right)\right)=I^{\sharp}\left(x_{\delta}\right)$ and hence $I_{0}(F) \leq \liminf _{\delta} I^{\sharp}\left(x_{\delta}\right)=I^{\sharp}(x)$.

$[\mathbf{2} \Rightarrow(\mathbf{4 . 3})]$ Here the proof is as before because in metric spaces we can find a sequence $\left\{x_{n}\right\} \subseteq H_{F}$, with $x=\lim x_{n}$.

Corollary 4.8 Assume that $G$ is a measurable function. If $F_{I^{\sharp}} \subseteq K_{G} \cup C_{I^{\sharp}}$, then $\left\{\left(X_{n}, G\left(X_{n}\right)\right)\right\}$ satisfies the large deviation principle upper bound with rate function $I_{0}$. 
Proof: It is enough to prove that $F_{I^{\sharp}} \subseteq K_{G} \cup C_{I^{\sharp}}$ implies the hypothesis of Theorem 4.7. Let $x \in F_{I^{\sharp}}$ and let $x_{\gamma}$ be a net converging to $x$. If $x \in K_{G}$, then there is a subnet $x_{\delta}$ along which $G$ converges to some point $y \in \mathcal{Y}$. If $x \in C_{I^{\sharp}}$, then the same net $x_{\gamma}$ satisfies $I^{\sharp}(x)=\lim _{\gamma} I^{\sharp}\left(x_{\gamma}\right)$.

Remark 4.9 The hypothesis of Corollary 4.5 implies that $F_{I^{\sharp}} \subseteq K_{G} \cup C_{I^{\sharp}}$, hence, the hypothesis of Corollary 4.5 implies not only a large deviation principle lower bound but also a large deviation principle for $\left\{\left(X_{n}, G\left(X_{n}\right)\right)\right\}$.

Finally we have a theorem combining the upper and lower bound:

Theorem 4.10 Suppose that for each $x \in F_{I^{\sharp}}$, and $y \in G^{x}, G$ is almost compact at $x$ and there is a sequence $\left\{x_{n}\right\}$ such that $x_{n} \rightarrow x, G\left(x_{n}\right) \rightarrow y, I^{\sharp}\left(x_{n}\right) \rightarrow I^{\sharp}(x)$ and for all $n, G$ is continuous at $x_{n}$. Then a large deviation principle holds for $\left\{\left(X_{n}, G\left(X_{n}\right)\right)\right\}$ with rate function $I_{0}$.

In particular, $\left\{G\left(X_{n}\right)\right\}$ satisfies the large deviation principle with rate function I given by:

$$
I(y)=\inf \left\{I_{0}(x, y): y \in G^{x}\right\}
$$

While in Theorem 1.1 the rate function is good, in Theorem 4.10 we do not know if this is the case. The results from the next section will allow us to conclude the goodness of the rate function.

Remark 4.11 If $G$ is continuous, then $G^{x}$ is the singleton $\{G(x)\}$ and we can take $x_{n}$ identically $x$. Therefore Theorem 4.10 implies the usual contraction principle.

Proof of Corollary 1.2: Cadlag or caglad functions are almost compact and quasicontinuous. The hypotheses imply that $F_{I^{\sharp}} \subseteq C_{G} \bigcup\left(C_{I^{\sharp}} \bigcap Q_{G}\right)$. Therefore the result follows. 
Proof of Corollary 1.3: As before, cadlag functions are almost compact, we just apply Theorem 4.7.

Proof of Corollary 1.4: Let $x$ with $I^{\sharp}(x)<\infty$. Take $i$ with $x \in A_{i}$.

- If $x \in A_{i}^{\circ}$, then $f$ is continuous at $x$.

- If $x \notin A_{i}^{\circ}$, then $x \in A_{i}-A_{i}^{\circ} \subseteq \partial A_{i}$, and $I^{\sharp}$ is continuous at $x$. We will prove that $f$ is quasi-continuous at $x$. Let $n>0$. Since $x \in A_{i} \subseteq \overline{A_{i}}=\overline{A_{i}^{\circ}}$, then there is $x_{n} \in$ $A_{i}^{\circ} \cap B_{1 / n}(x)$, therefore $f$ is continuous at $x_{n}$. Finally $f\left(x_{n}\right)=f_{i}\left(x_{n}\right) \longrightarrow f_{i}(x)=f(x)$, therefore $f$ is quasi-continuous at $x$.

Therefore $F_{I^{\sharp}} \subseteq C_{f} \bigcup\left(C_{I^{\sharp}} \bigcap Q_{f}\right)$, which is a subset of $K_{f} \bigcup C_{I^{\sharp}}$, and Theorem 4.4 and Theorem 4.7 apply.

\section{Almost Compactness and Goodness}

In this section we will not assume anything about large deviation principles. We work here with a general non-negative lower semicontinuous function $I^{\sharp}, G$ any function from $\mathcal{X}$ to $\mathcal{Y}$, and $I_{0}$ as defined in the beginning of the previous section. We study the relationship between almost compactness and the goodness of the rate function.

We remark that if $\mathcal{X}$ and $\mathcal{Y}$ are metric, we can replace "nets" by "sequences" in the definition of $G^{x}$

Theorem 5.1 Let $\mathcal{X}, \mathcal{Y}$ be metric spaces. If $G$ is almost compact at each $x \in F_{I^{\sharp}}$ and $I^{\sharp}$ is good, then $I_{0}$ is good.

Proof: Let $\left\{\left(x_{n}, y_{n}\right)\right\}$ be a sequence in $I_{0}^{-1}(-\infty, M]$. Since $I_{0}\left(x_{n}, y_{n}\right) \leq M<\infty, y_{n} \in G^{x_{n}}$. Since $I_{0}\left(x_{n}, y_{n}\right)=I^{\sharp}\left(x_{n}\right) \leq M$ and $I^{\sharp}$ is good, there is a subsequence $\left\{x_{n_{k}}\right\}$ of $\left\{x_{n}\right\}$ that converges to some $x$. Note that $I^{\sharp}(x)<\infty$ because $I^{\sharp}(x) \leq \liminf I^{\sharp}\left(x_{n}\right) \leq M$. Since $y_{n_{k}} \in$ $G^{x_{n_{k}}}$, by definition, there is a sequence $\left\{x_{n_{k}}^{m}\right\}_{m}$ converging to $x_{n_{k}}$ such that $G\left(x_{n_{k}}^{m}\right) \stackrel{m \rightarrow \infty}{\longrightarrow} y_{n_{k}}$. 
Now take $m_{k}$ with $x_{n_{k}}^{m_{k}} \in B_{1 / k}\left(x_{n_{k}}\right), G\left(x_{n_{k}}^{m_{k}}\right) \in B_{1 / k}\left(y_{n_{k}}\right)$. We know that $x_{n_{1}}^{m_{1}}, x_{n_{2}}^{m_{2}}, \ldots \rightarrow x$ and $I^{\sharp}(x)<\infty$ Using the almost compactness of $G$, we have that there is a subsequence: $x_{n_{k_{1}}}^{m_{k_{1}}}, x_{n_{k_{2}}}^{m_{k_{2}}}, \ldots$ of the previous one such that $G\left(x_{n_{k_{1}}}^{m_{k_{1}}}\right), G\left(x_{n_{k_{2}}}^{m_{k_{2}}}\right), \ldots$ converges to some $y$. We note that $y \in G^{x}$ and hence $I_{0}(x, y)=I^{\sharp}(x)$.

We note that

$$
d\left(x_{n_{k_{i}}}, x\right) \leq d\left(x_{n_{k_{i}}}, x_{n_{k_{i}}}^{m_{k_{i}}}\right)+d\left(x_{n_{k_{i}}}^{m_{k_{i}}}, x\right) \leq \frac{1}{k_{i}}+d\left(x_{n_{k_{i}}}^{m_{k_{i}}}, x\right)
$$

and similarly

$$
d\left(y_{n_{k_{i}}}, y\right) \leq d\left(y_{n_{k_{i}}}, G\left(x_{n_{k_{i}}}^{m_{k_{i}}}\right)\right)+d\left(G\left(x_{n_{k_{i}}}^{m_{k_{i}}}\right), y\right) \leq \frac{1}{k_{i}}+d\left(G\left(x_{n_{k_{i}}}^{m_{k_{i}}}\right), y\right)
$$

Therefore $\left(x_{n_{k_{i}}}, y_{n_{k_{i}}}\right) \longrightarrow(x, y)$ which means that $I_{0}$ is good.

Definition 5.2 We say that a $\mathbb{R}$-valued function $f$ is locally bounded if for each $x$ in its domain, there is $M$ and a neighborhood $\mathcal{U}$ of $x$ such that $\sup _{u \in \mathcal{U}} f(u) \leq M$.

Theorem 5.3 If $I_{0}$ is good and $I^{\sharp}$ is locally bounded, then $G$ is almost compact at each $x$ in $F_{I^{\sharp}}$.

Proof: Let $x \in F_{I^{\sharp}}$ and $x_{\alpha}$ with $x_{\alpha} \rightarrow x$. Since $I^{\sharp}$ is locally bounded, we can find a subnet $\left\{x_{\beta}\right\}$ of $\left\{x_{\alpha}\right\}$ such that $M \equiv \lim I^{\sharp}\left(x_{\beta}\right)<\infty$.

Select $\beta_{0}$ so that $\beta \geq \beta_{0}$ implies $I^{\sharp}\left(x_{\beta}\right) \leq M+1$. Hence for $\beta \geq \beta_{0}$ we have $I_{0}\left(x_{\beta}, G\left(x_{\beta}\right)\right)=$ $I^{\sharp}\left(x_{\beta}\right) \leq M+1$. Since $I_{0}$ is good we have that there is a subnet $\left\{\left(x_{\gamma}, G\left(x_{\gamma}\right)\right)\right\}$ of $\left\{\left(x_{\beta}, G\left(x_{\beta}\right)\right)\right\}_{\beta \geq \beta_{0}}$ such that $x_{\gamma} \rightarrow x$ and $G\left(x_{\gamma}\right) \rightarrow y$. Hence $G$ is almost compact. 
Proposition 5.4 If $I_{0}$ is good, then $I^{\sharp}$ is good.

Proof: We know that $\{(x, y): I(x, y) \leq M\}$ is compact on the product space. Projecting on the first coordinate is a continuous function and continuous maps take compact sets into compact sets. Therefore this implies the compactness of the level set $\left\{x: I^{\sharp}(x) \leq M\right\}$.

From the above theorems we know that if $I^{\sharp}$ is good, and locally bounded on $F_{I^{\sharp}}$, then : $G$ is almost compact $\Leftrightarrow I_{0}$ is good. Or if you prefer: If $I^{\sharp}$ is locally bounded on $F_{I^{\sharp}}$, then $I_{0}$ is $\operatorname{good} \Leftrightarrow I^{\sharp}$ is good and $G$ is almost compact.

Remark 5.5 Assume $\left\{X_{n}\right\}$ satisfies the large deviation principle with a rate function $I^{\sharp}$ locally bounded on $F_{I^{\sharp}}$. Define

$$
I_{0}(x, y)=\left\{\begin{array}{rll}
I^{\sharp}(x) & : & y \in G^{x} \\
\infty & : & \text { otherwise }
\end{array} .\right.
$$

1. If $\left\{X_{n}, G\left(X_{n}\right)\right\}$ satisfies the large deviation principle upper bound with good rate function $I_{0}$, then $G$ is almost compact at each $x \in F_{I^{\sharp}}$ and $I^{\sharp}$ is good

2. Conversely, if $G$ is an almost compact function at each $x \in F_{I^{\sharp}}$ and $I^{\sharp}$ is good, then $\left\{X_{n}, G\left(X_{n}\right)\right\}$ satisfies the large deviation principle upper bound with good rate function $I_{0}$

Another observation is that quasi-continuity does not imply almost compactness; take the indicator $(-\infty, 0]$ plus the product of $1 / x$ with the indicator of $(0, \infty)$

Almost compact does not imply quasi-continuous. Simply take the function that is zero at zero and $\operatorname{sign}(x)$ everywhere else.

It is false that if a function is almost compact, then it is measurable. Take the indicator of a non-measurable set.

Finally we have the following observation: 
Proposition 5.6 If $G$ is almost compact and $I_{0}$ is locally bounded on $F_{I_{0}}$, then $I^{\sharp}$ is locally bounded on $F_{I^{\sharp}}$.

Proof: Let $x$ satisfy $I^{\sharp}(x)<\infty$, and let $\left\{x_{\alpha}\right\}$ be a net converging to $x$. Then there is a subnet $\left\{x_{\beta}\right\}$ of $\left\{x_{\alpha}\right\}$ with $G\left(x_{\beta}\right) \rightarrow y$ for some $y$ and $y \in G^{x}$. Then $I_{0}(x, y)=I^{\sharp}(x)<\infty$. Since $\left(x_{\beta}, G\left(x_{\beta}\right)\right)$ converges to $(x, y)$ and $I_{0}$ is locally bounded on $F_{I_{0}}$, there is a subnet $\left\{\left(x_{\gamma}, G\left(x_{\gamma}\right)\right)\right\}$ for which $\infty>\lim _{\gamma} I_{0}\left(x_{\gamma}, G\left(x_{\gamma}\right)\right)=\lim _{\gamma} I^{\sharp}\left(x_{\gamma}\right)$, i.e. we have a subnet $\left\{x_{\gamma}\right\}$ of $\left\{x_{\alpha}\right\}$ with $\lim _{\gamma} I^{\sharp}\left(x_{\gamma}\right)<\infty$, and hence $I^{\sharp}$ is locally bounded on $F_{I^{\sharp}}$.

\section{Almost Compactness and Exponential Tightness}

We assume in all this section the following situation: $\Omega \stackrel{X_{n}}{\longrightarrow} \mathcal{X} \stackrel{G}{\longrightarrow} \mathcal{Y}$. We study here equivalences of almost compactness and some relations between exponential tightness and almost compactness.

Lemma 6.1 Consider the following statements:

1. $G$ is almost compact.

2. If $K$ is compact in $\mathcal{X}, \overline{G(K)}$ is compact in $\mathcal{Y}$.

3. For any $x \in \mathcal{X}$ and any sequence converging to $x$ there is a subsequence along which $G$ converges. ( $G$ is almost compact by sequences)

Then $\mathbf{2} \Rightarrow \mathbf{3}$. If $\mathcal{Y}$ is metric $\mathbf{3} \Rightarrow \mathbf{2}$. If $\mathcal{Y}$ has a countable basis at each of its points, then $\mathbf{1} \Rightarrow \mathbf{3}$. If $\mathcal{X}$ and $\mathcal{Y}$ are metric, then $\mathbf{3} \Rightarrow \mathbf{1}$. If $\mathcal{X}$ is locally compact $\mathbf{2} \Rightarrow \mathbf{1}$. In general $\mathbf{1}$ $\supsetneqq \mathbf{2}$ (and therefore, in general $\mathbf{3} \supsetneqq \mathbf{2}$ ). In general $\mathbf{3} \supsetneqq \mathbf{1}$ and $\mathbf{1} \supsetneqq \mathbf{3}$. 


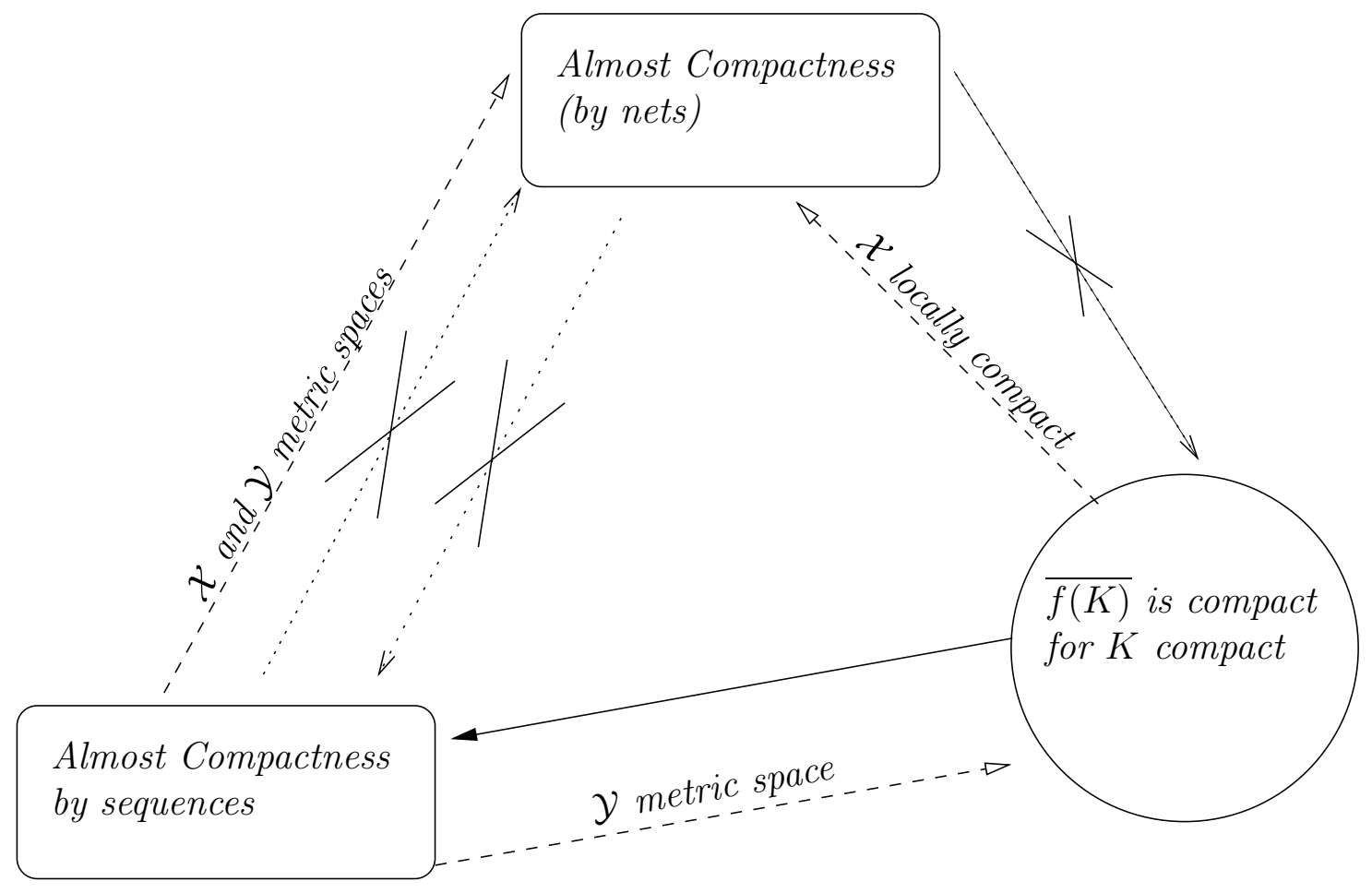

Fig 1.- Relations of Theorem 6.1 can be graphically appreciated.

\section{Proof.}

$[\mathbf{2} \Rightarrow \boldsymbol{3}]$ Given a sequence $\left\{x_{n}\right\}$ converging to $x$, the set $K=\left\{x_{n}: n \geq 1\right\} \cup\{x\}$ is compact, by hypothesis, $J=\overline{G(K)}$ is compact, $J$ contains the net $G\left(x_{n}\right)$, then it has a converging subnet, again, by the same argument as before, we can find a subsequence of $\left\{x_{n}\right\}$ along which $G$ converges.

$[\mathbf{1} \Rightarrow \boldsymbol{3}]$ (If $\mathcal{Y}$ has a countable basis at each of its points) Let $\left\{x_{n}\right\}$ be a sequence converging to $x$. Since $G$ is almost compact, the sequence $\left\{G\left(x_{n}\right)\right\}$ has a cluster point $y$ in $\mathcal{Y}$, since $\mathcal{Y}$ has a countable basis at $y$, there is a subsequence of $\left\{G\left(x_{n}\right)\right\}$ converging to $y$. (See $[6$, Theorem 8-c, P72])

$[3 \Rightarrow 2]$ (If $\mathcal{Y}$ is metric) Let $J=\overline{G(K)}$. We will prove that $J$ is compact. Let $\left\{z_{n}\right\}$ be a sequence in $J$. Then there are sequences $\left\{y_{n}\right\}$ in $G(K),\left\{x_{n}\right\}$ in $K$ such that 
$d\left(z_{n}, y_{n}\right) \rightarrow 0, y_{n}=G\left(x_{n}\right)$. Since $\left\{x_{n}\right\} \subseteq K$ and $K$ is compact, there is a subsequence $\left\{x_{n^{\prime}}\right\}$ of $\left\{x_{n}\right\}$ such that $x_{n^{\prime}} \rightarrow x$. Since $G$ is almost compact, there is a subsequence $\left\{x_{n^{\prime \prime}}\right\}$ of $\left\{x_{n^{\prime}}\right\}$ such that $G\left(x_{n^{\prime \prime}}\right)$ converges to some $y$. But $\left\{y_{n^{\prime \prime}}=G\left(x_{n^{\prime \prime}}\right)\right\} \rightarrow y$ and $d\left(z_{n^{\prime \prime}}, y_{n^{\prime \prime}}\right) \rightarrow 0$, hence $z_{n^{\prime \prime}} \rightarrow y$. Since $\left\{z_{n^{\prime \prime}}\right\}$ is a subsequence of $\left\{z_{n}\right\}$ and $J$ is closed, $y \in J$. This shows that $J$ is compact.

$[\mathbf{3} \Rightarrow \mathbf{1}]$ (If $\mathcal{X}$ and $\mathcal{Y}$ are metric) First we note that $G^{x}$ is compact: Let $\left\{y_{n}\right\}$ be a sequence in $G^{x}$, then for each $n$ there is sequence $\left\{x_{n}^{k}\right\}_{k}$ converging to $x$ as $k \rightarrow \infty$ such that $G\left(x_{n}^{k}\right)$ converges to $y_{n}$ as $k \longrightarrow \infty$. Let $k_{n}$ such that $d\left(x, x_{n}^{k_{n}}\right), d\left(G\left(x_{n}^{k_{n}}\right), y_{n}\right)<1 / n$. By hypothesis, the sequence $\left\{x_{n}^{k_{n}}\right\}$ has a subsequence $\left\{x_{n^{\prime}}^{k_{n^{\prime}}}\right\}$ along which $G$ converges to some $y \in \mathcal{Y}$. Hence the corresponding subsequence $\left\{y_{k_{n^{\prime}}}\right\}$ of $\left\{y_{n}\right\}$ converges to $y$, as $n \rightarrow \infty$, therefore $y \in G^{x}$, and then $G^{x}$ is compact and closed.

Let $\left\{x_{\alpha}\right\}_{\alpha \in A} \longrightarrow x$. For each $n$, there is $\alpha_{n}$ such that if $\alpha>\alpha_{n}$, then $G\left(x_{\alpha}\right) \in B_{n} \equiv$ $\left\{y: d\left(y, G^{x}\right)<1 / n\right\}$ (otherwise we can find a sequence $\left\{x_{n}\right\}$ converging to $x$ along which $d\left(G\left(x_{n}\right), G^{x}\right) \geq 1 / n$, which is impossible.)

For each $\alpha \in A$ choose $y_{\alpha} \in G^{x}$ with $d\left(G\left(x_{\alpha}\right), y_{\alpha}\right)=d\left(G\left(x_{\alpha}\right), G^{x}\right)$. Since $G^{x}$ is compact, the net $\left\{y_{\alpha}\right\}$ has a subnet $\left\{y_{\beta}\right\}$ converging to some $y \in \mathcal{Y}$. If $\beta>\alpha_{n}$, then $d\left(G\left(x_{\beta}\right), y\right) \leq d\left(G\left(x_{\beta}\right), y_{\beta}\right)+d\left(y_{\beta}, y\right) \leq 1 / n+d\left(y_{\beta}, y\right)$. Hence $\left\{G\left(x_{\beta}\right)\right\}$ converges to $y$.

$[\mathbf{2} \Rightarrow \mathbf{1}]$ (If $\mathcal{X}$ is locally compact) Take $x_{\alpha} \longrightarrow x, U$ a compact neighborhood of $x$, then there is $\alpha_{0}$ such that if $\alpha \geq \alpha_{0}$, then $x_{\alpha} \in U$, therefore the compact set $\overline{G(U)}$ contains the net $\left\{x_{\alpha}\right\}_{\alpha \geq \alpha_{0}}$, therefore it has a converging subnet, which is a subnet of the original.

$[\mathbf{3} \neq \mathbf{1}$,$] Let \Omega$ be the first non-countable ordinal and $[0, \Omega]$ to be the set of ordinals between 0 and $\Omega$, consider the topology generated by the sets $(a, b)$, the set of ordinals greater than $a$ and smaller that $b$. Here just take $G:[0, \Omega] \longrightarrow[0, \Omega)$ and $G(x)=x$ for $x \neq \Omega$, and $G(\Omega)=0$. Then any sequence in $[0, \Omega)$ has a convergent subsequence 
$\left([0, \Omega)\right.$ is sequentially compact) but given the net $\left\{x_{\alpha}=\alpha\right\}_{\alpha \in[0, \Omega)}$ that converges to $\Omega$ there is no subnet of $G\left(x_{\alpha}\right)=\alpha$ that converges in the range of $G$.

$[\mathbf{1} \supsetneqq \mathbf{3}$,$] Take a compact space \mathcal{Y}$ that is not sequentially compact and a sequence $\left\{a_{n}\right\}$ in $\mathcal{Y}$ that has no convergent subsequence. Take a sequence $\left\{x_{n}\right\}$ in $\mathcal{Y}$ converging to a point $x$ such that all the $x_{n}^{\prime} s$ are different. Define a function $G$ from $\mathcal{Y}$ to $\mathcal{Y}$ by $G(x)=a_{n}$ for $x=x_{n}$ and $G(x)=a_{1}$ for $x \neq x_{1}, x_{2}, \ldots$ The function $G$ is certainly almost compact because $\mathcal{Y}$ is compact, but it is not almost compact by sequences because the sequence $x_{n}$ converges to $x$ but the sequence $\left\{G\left(x_{n}\right)\right\}$ has no converging subsequence.

$[\mathbf{1} \supsetneqq \mathbf{2}$,$] This proof deserves to be done separately in Remark 6.2.$

From the above theorem, we can see that in the case of metric spaces all of the above are equivalent. If $\mathcal{X}$ is locally compact and $\mathcal{Y}$ is metric, all of the above are equivalent. This happens if $\mathcal{X}$ and $\mathcal{Y}$ are Euclidian spaces, for example.

Remark 6.2 If $K$ is compact and $G$ is almost compact, $\overline{G(K)}$ is not necessarily compact.

\section{Proof:}

Take $\mathcal{X}=\mathbb{R} \times[0, \infty)$ with the usual topology, $\mathcal{Y}=\mathbb{R} \times[0, \infty)$ with the following topology: A basic is a ball inside of $\mathbb{R} \times(0, \infty)$ or a basic is $C_{\epsilon}^{x}$ : the upper bound of a ball with center on the $x$ axis minus its diameter plus its center, i.e.

$$
C_{\epsilon}^{x}=\left\{(u, v) \in B_{\epsilon}(x, 0):(u, v)=(x, 0) \text { or } v>0\right\} .
$$

Note that $\mathcal{Y}$ is a Hausdorff space that is not regular. Define $G$ by:

$$
G(x, y)=\left\{\begin{array}{lll}
(x, y) & : & y>0 \\
(0,0) & : & y=0
\end{array}\right.
$$


Then $G$ is an almost compact function and if you take $K=[1,2] \times[0,1]$, then $\overline{G(K)}=$ $[1,2] \times[0,1] \cup\{(0,0)\}$ which is not compact in this strange topology because it has an infinite number of points on the $x$-axis, and compact sets here have at most finitely many points in the $x$-axis, otherwise, for example, $[3,4] \times\{0\}$ is contained in the union of $C_{\epsilon}^{x}$ for $x \in[3,4]$, but there is no finite union that covers that set.

Theorem 6.3 If $\left\{X_{n}\right\}$ is exponentially tight, $\mathcal{Y}$ is a metric space and $G$ is almost compact, then $\left\{G\left(X_{n}\right)\right\}$ is exponentially tight.

Proof. Let $\alpha>0$. Then there is a compact set $J$ such that:

$$
\varlimsup_{n} \frac{1}{n} \log P\left[X_{n} \notin J\right] \leq-\alpha / 2
$$

From Lemma $6.1, K=\overline{G(J)}$ is compact.

$$
\begin{aligned}
\liminf \frac{1}{n} \log P\left[G\left(X_{n}\right) \notin K\right] & =\liminf \frac{1}{n} \log P\left[X_{n} \notin G^{-1}(K)\right] \\
& \leq \liminf \frac{1}{n} \log P\left[X_{n} \notin J\right] \\
& \leq-\alpha
\end{aligned}
$$

where the first inequality follows from $G^{-1}(K)=G^{-1}(\overline{G(J)}) \supseteq J$. Consequently $\left\{G\left(X_{n}\right)\right\}$ is exponentially tight.

Remark 6.4 If $\left\{\mu_{n}\right\}$ is exponentially tight and $G$ is almost compact, $\left\{\mu_{n} \circ G^{-1}\right\}$ is not necessarily exponentially tight.

\section{Proof:}

Take $\mathcal{X}$ and $\mathcal{Y}$ as in the previous remark provided with those topologies. Define $G$ by:

$$
G(x, y)=\left\{\begin{aligned}
(x, y) & : y>0, \\
(1,1) & : y=0, x \in(-\infty, 0] \cup(1, \infty) . \\
\left(\frac{1}{n}, x-\frac{1}{n}\right) & : y=0, x \in\left(\frac{1}{n}, \frac{1}{n-1}\right] .
\end{aligned}\right.
$$


Then $G$ is an almost compact function that is not continuous. Consider:

$$
\begin{aligned}
& A_{1}=\left(\frac{1}{2}, 1\right] \\
& A_{2}=\left(\frac{1}{2}, \frac{1}{2}+\frac{1}{6}\right] \quad A_{3}=\left(\frac{1}{3}, \frac{1}{2}\right] \\
& A_{4}=\left(\frac{1}{2}, \frac{1}{2}+\frac{1}{12}\right] \quad A_{5}=\left(\frac{1}{3}, \frac{1}{3}+\frac{1}{12}\right] \quad A_{6}=\left(\frac{1}{4}, \frac{1}{3}\right] \\
& A_{7}=\left(\frac{1}{2}, \frac{1}{2}+\frac{1}{20}\right] \quad A_{8}=\left(\frac{1}{3}, \frac{1}{3}+\frac{1}{20}\right] \quad A_{9}=\left(\frac{1}{4}, \frac{1}{4}+\frac{1}{20}\right] \quad A_{10}=\left(\frac{1}{5}, \frac{1}{4}\right]
\end{aligned}
$$

Let $m_{n}$ be supported on $A_{n}$, and let $\mu_{n}(B)=m_{n}\{x:(x, 0) \in B\}$.

Claim $\left\{\mu_{n} \circ G^{-1}\right\}$ is not exponentially tight.

Proof:(claim) Assume the opposite. Then there is a compact set $K$ such that

$$
\varlimsup_{n} \frac{1}{n} \log \mu_{n} \circ G^{-1}\left(K^{c}\right) \leq-3 .
$$

$K$ has a finite number of points on the $x$-axis. Let $x_{0}$ be the smallest positive number such that $\left(x_{0}, 0\right) \in K$. If $K$ does not have a point on the $x$-axis, take $x_{0}=2$. Take $n$ with $\frac{1}{n}<x_{0}$. Since $\left(\frac{1}{n}, 0\right) \in K^{c}$ and $K^{c}$ is open (we are in a Hausdorff space), there is $\epsilon \in\left(0, \frac{1}{n}\right)$ with $C_{\epsilon}^{1 / n} \subseteq K^{c}$. We can take $\epsilon=\frac{1}{m(m-1)}$ for $m$ big enough. Therefore $\frac{1}{n} \times\left(0, \frac{1}{m(m+1)}\right] \subseteq K^{c}$.

There is some $k$ such that $A_{k}=\left(\frac{1}{n}, \frac{1}{n}+\frac{1}{m(m+1)}\right]$ but $G\left(A_{k} \times\{0\}\right)=\frac{1}{n} \times\left(0, \frac{1}{m(m+1)}\right] \subseteq K^{c}$. Actually there is a sequence $k_{1}<k_{2}<\ldots$ with $A_{k_{i}}=\left(\frac{1}{n}, \frac{1}{n}+\frac{1}{(m+i)(m+i+1)}\right]$, of course $G\left(A_{k_{i}} \times\{0\}\right) \subseteq K^{c}$. Consider the subsequence $\left\{\mu_{k_{i}}\right\}$. Then

$$
\begin{aligned}
-3 & \geq \varlimsup_{k} \frac{1}{k} \log \mu_{k} \circ G^{-1}\left(K^{c}\right) \\
& \geq \varlimsup_{i} \frac{1}{k_{i}} \log \mu_{k_{i}} \circ G^{-1}\left(K^{c}\right) \\
& \geq \varlimsup_{i} \frac{1}{k_{i}} \log \mu_{k_{i}}\left(A_{k_{i}} \times\{0\}\right) \\
& =\varlimsup_{i} \frac{1}{k_{i}} \log m_{k_{i}}\left(A_{k_{i}}\right)=\varlimsup_{i} \frac{1}{k_{i}} \log 1=0,
\end{aligned}
$$

which is impossible.

The following theorem says that, under certain assumptions, it is enough to check the upper bound just on "closed boxes" instead of all closed sets. 
Theorem 6.5 If $\left\{\left(X_{n}, G\left(X_{n}\right)\right)\right\}$ satisfies the large deviation principle upper bound on "closed boxes" with rate function $I_{0}$ defined by 1.1 on a metric space, then $\left\{\left(X_{n}, G\left(X_{n}\right)\right)\right\}$ satisfies the large deviation principle upper bound and $I_{0}$ is good.

Proof: From Lemma 6.3, $\left\{G\left(X_{n}\right)\right\}$ is exponentially tight, hence $\left\{\left(X_{n}, G\left(X_{n}\right)\right)\right\}$ is exponentially tight. Therefore it is enough to prove the upper bound for $K$ compact.

For each $k>0$, let $F_{k}=\bigcup_{i=1}^{N_{k}} \overline{B_{1 / k}\left(x_{i}\right)} \times \overline{B_{1 / k}\left(y_{i}\right)}$ where $K \subseteq \bigcup_{i=1}^{N_{k}} B_{1 / k}\left(x_{i}\right) \times B_{1 / k}\left(y_{i}\right)$ for some $\left(x_{1}, y_{1}\right), \ldots,\left(x_{N_{k}}, y_{N_{k}}\right)$ in $K$. It is clear that $K=\bigcap_{k=1}^{\infty} F_{k}$. By hypothesis:

$$
\begin{aligned}
& \varlimsup \frac{1}{n} \log P\left[\left(X_{n}, G\left(X_{n}\right)\right) \in K\right] \leq \varlimsup \lim \frac{1}{n} \log P\left[\left(X_{n}, G\left(X_{n}\right)\right) \in F_{k}\right] \\
& \leq-I_{0}\left(F_{k}\right) \text {. }
\end{aligned}
$$

Therefore

$$
\begin{aligned}
& \varlimsup \frac{1}{n} \log P\left[\left(X_{n}, G\left(X_{n}\right)\right) \in K\right] \leq \inf _{k}-I_{0}\left(F_{k}\right) \\
& =-\sup _{k} I_{0}\left(F_{k}\right) \text {. }
\end{aligned}
$$

Theorem 5.1 assures that $I_{0}$ is good and any good function satisfies:

$$
\sup _{k} I_{0}\left(F_{k}\right)=I_{0}\left(\bigcap_{k=1}^{\infty} F_{k}\right)
$$

Then the large deviation principle upper bound holds.

Finally if we combine the upper and lower bound in the previous, theorem we obtain Theorem 1.1.

\section{$7 \quad$ An application of Theorem 1.1}

Many of the examples of rate functions in infinite dimensions are of the form

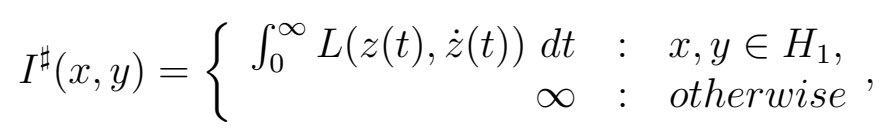


where $H_{1}$ is the subspace of the continuous functions on the interval $[0, \infty)$ with the topology of uniform convergence on bounded time intervals and $L$ is a function that is convex on the second entry. An example of this type of function is when $z=(x, y) \in \mathbb{R}^{d} \times \mathbb{R}^{d}$, and $L(x, y, \dot{x}, \dot{y})=\|\dot{x}(t)\|^{2}+\|\dot{y}(t)\|^{2}$. In this case the rate function $I^{\sharp}$ is the rate function for $\sqrt{\epsilon} W$, where $W$ is the Brownian motion. We will prove that for a sequence of processes $\left(X_{n}, Y_{n}\right)$ satisfying a large deviation principle with this rate function, a large deviation principle can be obtained for $G_{\epsilon}\left(X_{n}, Y_{n}\right)$ where $G_{\epsilon}$ is an $\epsilon$-approximation of the stochastic integral that will be defined next.

Let $\left\{\left(X_{n}, Y_{n}\right)\right\}$ be a sequence of random functions on $C_{0}\left([0, \infty), \mathbb{R}^{d}\right)$, (the set of continuous functions from $[0, \infty)$ to $\mathbb{R}^{d}$ that start at 0$)$ and whose derivative is in $L^{2}$. Assume that $\left\{\left(X_{n}, Y_{n}\right)\right\}$ satisfies the large deviation principle with rate function:

$$
I^{\sharp}(x, y)=\left\{\begin{array}{r}
\int_{0}^{\infty}\left[\|\dot{x}(t)\|^{2}+\|\dot{y}(t)\|^{2}\right] d t \quad: \quad x, y \in H_{1}, \\
\infty \quad: \quad \text { otherwise }
\end{array}\right.
$$

where $\|\cdot\|$ is the usual norm in $\mathbb{R}^{d}$ and $H_{1}=\left\{x \in C_{0}\left([0, \infty), \mathbb{R}^{d}\right): x\right.$ is abs. cont. , $\|\dot{x}\|^{2} \in$ $\left.L_{1}\right\}$. Consider the function $g_{\epsilon}$ defined on $C_{0}([0, \infty), \mathbb{R})$ by $g_{\epsilon}(x, y)=x^{\epsilon} \cdot y$ the $\epsilon$-approximation to the integral defined by: $\tau_{0}^{\epsilon} \equiv 0$,

$$
\begin{gathered}
\tau_{n}^{\epsilon}=\inf \left\{t>\tau_{n-1}^{\epsilon}:\left|x(t)-x\left(\tau_{n-1}^{\epsilon}\right)\right|>\epsilon\right\} \\
g_{\epsilon}(x, y)(t)=\sum_{i=0}^{\infty} x\left(\tau_{i-1}^{\epsilon}\right)\left(y\left(\tau_{i}^{\epsilon} \wedge t\right)-y\left(\tau_{i-1}^{\epsilon} \wedge t\right)\right) .
\end{gathered}
$$

$g_{\epsilon}$ represents the integral of a piecewise constant function $x^{\epsilon}$ with respect to $y$, so it is the Lebesgue-Stieltjes integral. Define $G_{\epsilon}: H_{1} \times H_{1} \longrightarrow C_{0}\left([0, \infty), \mathbb{R}^{d}\right)$ by

$$
G_{\epsilon}(x, y)=\left(g_{\epsilon}\left(x_{1}^{\epsilon}, y_{1}\right), \ldots, g_{\epsilon}\left(x_{d}^{\epsilon}, y_{d}\right)\right)
$$

where $x=\left(x_{1}, \ldots, x_{d}\right), y=\left(y_{1}, \ldots, y_{d}\right)$.

The goal is to prove a large deviation principle for $\left(X_{n}, Y_{n}, G_{\epsilon}\left(X_{n}, Y_{n}\right)\right)$. In a paper soon to appear $\left(\left[5\right.\right.$, Theorem 2.1]), we prove that $G_{\epsilon}$ is an almost compact function.

We introduce and prove the following lemmas that will be useful. 
Lemma 7.1 In a metric space, an almost compact function $F$ is continuous at $x$ if and only if $F^{x}=\{F(x)\}$.

Proof: It is clear that if $F$ is continuous $F^{x}=\{F(x)\}$. Conversely, assume that $F^{x}=$ $\{F(x)\}$. Let $\left\{x_{n}\right\}$ be a sequence converging to $x$. Using the almost compactness of $F$, we can find a subsequence $\left\{x_{n^{\prime}}\right\}$ converging to $x$ along which $F$ converges to some $y$. Then $y \in F^{x}$, i.e. $y=F(x)$. Hence $F$ is continuous at $x$.

Lemma 7.2 Let $x, y \in C_{0}\left([0, \infty), \mathbb{R}^{d}\right), z \in G_{\epsilon}^{(x, y)}$. Then, for each $k \in\{1,2, \ldots, d\}$ there is a sequence $\left\{r_{i}^{k}\right\}_{i}$ satisfying:

1. $\left\{r_{i}^{k}\right\} \subseteq[0, \infty], \quad r_{0}^{k}=0, \quad\left\{r_{i}^{k}\right\}_{i} \uparrow \infty$

2. For each $k \in\{1 \ldots d\}$ and for all $t$ in $\left[r_{i}^{k}, r_{i+1}^{k}\right),\left|x(t)-x\left(r_{i}^{k}\right)\right| \leq \epsilon$

3. For each $k \in\{1 \ldots d\}$ and for all $i,\left|x\left(r_{i}^{k}\right)-x\left(r_{i+1}^{k}\right)\right|=\epsilon$

such that for each $k \in\{1,2, \ldots, d\}$ :

$$
z^{k}(t)=\sum_{i=1}^{j} x^{k}\left(r_{i-1}^{k}\right)\left(y^{k}\left(r_{i}^{k}\right)-y^{k}\left(r_{i-1}^{k}\right)\right)+x^{k}\left(r_{j}^{k}\right)\left(y^{k}(t)-y^{k}\left(r_{j}^{k}\right)\right), \quad t \in\left[r_{j}^{k}, r_{j+1}^{k}\right) .
$$

Proof: Since $z \in G_{\epsilon}^{(x, y)}$. Then there is a sequence $\left\{\left(x_{n}, y_{n}\right)\right\}$ converging to $(x, y)$ such that $G_{\epsilon}\left(x_{n}, y_{n}\right)$ converges to $z$ uniformly on bounded time intervals.

Let $k \geq 1$ be fixed. Since $\tau_{1}^{\epsilon}\left(x_{n}^{k}\right)<\tau_{2}^{\epsilon}\left(x_{n}^{k}\right)$ we have $\liminf _{n \rightarrow \infty} \tau_{1}^{\epsilon}\left(x_{n}^{k}\right) \leq \liminf _{n \rightarrow \infty} \tau_{2}^{\epsilon}\left(x_{n}^{k}\right)$. Using a diagonal argument, we can select $\left\{x_{n^{\prime}}^{k}\right\}_{n^{\prime}}$ a subsequence of $\left\{x_{n}^{k}\right\}_{n}$ such that for all $m \geq 1, \tau_{k}^{\epsilon}\left(x_{n^{\prime}}^{k}\right) \stackrel{n^{\prime} \rightarrow \infty}{\longrightarrow} r_{m}^{k}$ and $r_{1}^{k} \leq r_{2}^{k} \leq \ldots \leq r_{m}^{k} \leq \ldots$ Since $x, y$ are continuous

$$
\left(x_{n^{\prime}}^{k}\left(\tau_{k}^{\epsilon}\left(x_{n^{\prime}}^{k}\right)\right), y_{n^{\prime}}^{k}\left(\tau_{k}^{\epsilon}\left(x_{n^{\prime}}^{k}\right)\right)\right) \stackrel{n^{\prime} \rightarrow \infty}{\longrightarrow}\left(x^{k}\left(r_{m}^{k}\right), y^{k}\left(r_{m}^{k}\right)\right) .
$$

Let's rename the subsequence $\left\{\left(x_{n^{\prime}}^{k}, y_{n^{\prime}}^{k}\right)\right\}$ by $\left\{\left(x_{n}^{k}, y_{n}^{k}\right)\right\}$. 
To prove that $r_{i}^{k} \uparrow \infty$ as $i \rightarrow \infty$, suppose now that for all $n, r_{n}^{k}<\infty$ and suppose that $r_{n}^{k} \uparrow$ $R$, where $R<\infty$. The facts that $R<\infty$ and $x_{n}^{k} \rightarrow x^{k}$ imply that $\lim _{m \rightarrow \infty} x^{k}\left(r_{m}^{k}\right)=x^{k}(R)$. Since $\epsilon \leq\left|x_{n}^{k}\left(\tau_{m}^{\epsilon}\left(x_{n}^{k}\right)\right)-x_{n}^{k}\left(\tau_{m+1}^{\epsilon}\left(x_{n}^{k}\right)\right)\right|$, we obtain $\epsilon \leq \lim _{n}\left|x_{n}^{k}\left(\tau_{m}^{\epsilon}\left(x_{n}^{k}\right)\right)-x_{n}^{k}\left(\tau_{m+1}^{\epsilon}\left(x_{n}^{k}\right)\right)\right|=$ $\left|x^{k}\left(r_{m}^{k}\right)-x\left(r_{m+1}^{k}\right)\right|$, taking limits in the previous inequality as $m$ goes to infinity and we obtain that $\epsilon \leq\left|x^{k}(R)-x^{k}(R)\right|$, which is impossible. Therefore assuming that $R<\infty$ leads us to a contradiction. Therefore

- $\left(x_{n}^{k}, y_{n}^{k}\right) \stackrel{n \rightarrow \infty}{\longrightarrow}\left(x^{k}, y^{k}\right), \quad\left\{r_{i}^{k}\right\}_{i} \subseteq[0, \infty], \quad r_{0}^{k}=0, \quad\left\{r_{i}^{k}\right\}_{i} \uparrow \infty$

- $\tau_{i}^{\epsilon}\left(x_{n}^{k}\right) \stackrel{n \rightarrow \infty}{\longrightarrow} r_{i}^{k}$

- $\left(x_{n}^{k}\left(\tau_{m}^{\epsilon}\left(x_{n}^{k}\right)\right), y_{n}^{k}\left(\tau_{m}^{\epsilon}\left(x_{n}^{k}\right)\right)\right) \stackrel{n \rightarrow \infty}{\longrightarrow}\left(x^{k}\left(r_{m}^{k}\right), y^{k}\left(r_{m}^{k}\right)\right)$

Since $g_{\epsilon}\left(x_{n}^{k}, y_{n}^{k}\right) \stackrel{n \rightarrow \infty}{\longrightarrow} z^{k}$, we conclude that

$$
z^{k}(t)=\sum_{i=1}^{j} x^{k}\left(r_{i-1}^{k}\right)\left(y^{k}\left(r_{i}^{k}\right)-y^{k}\left(r_{i-1}^{k}\right)\right)+x^{k}\left(r_{j}^{k}\right)\left(y^{k}(t)-y^{k}\left(r_{j}^{k}\right)\right), \quad t \in\left[r_{j}^{k}, r_{j+1}^{k}\right) .
$$

To finish the proof of this lemma we will only need to prove items (2) and (3) from Lemma (7.2):

Let $t$ be in $\left[r_{i}^{k}, r_{i+1}^{k}\right)$. Let $\delta>0$ such that $t \in\left[r_{i}^{k}, r_{i}^{k}+\delta\right)$ implies $\left|x^{k}(t)-x^{k}\left(r_{i}^{k}\right)\right|<\epsilon$. Consider now $t \in\left[r_{i}^{k}+\delta, r_{i+1}^{k}\right)$, and let $\eta$ be any positive number. There exists $N$ such that if $n \geq N$, then $\left|x^{k}(t)-x_{n}^{k}(t)\right|<\eta / 2,\left|x_{n}^{k}\left(\tau_{i}^{\epsilon}\left(x_{n}^{k}\right)\right)-x^{k}\left(r_{i}^{k}\right)\right|<\eta / 2,\left|\tau_{i+1}^{\epsilon}\left(x_{n}^{k}\right)-r_{i+1}^{k}\right|<\left|r_{i+1}^{k}-t\right|$ and $\left|\tau_{i}^{\epsilon}\left(x_{n}^{k}\right)-r_{i}^{k}\right|<\delta / 2$.

Therefore:

$$
\tau_{i}^{\epsilon}\left(x_{n}^{k}\right) \leq r_{i}^{k}+\delta / 2 \leq t=r_{i+1}^{k}-\left|r_{i+1}^{k}-t\right|<\tau_{i+1}^{\epsilon}\left(x_{n}^{k}\right)
$$

Consequently if we choose $n=N$ :

$$
\begin{aligned}
\left|x^{k}(t)-x^{k}\left(r_{i}^{k}\right)\right| & \leq\left|x^{k}(t)-x_{n}^{k}(t)\right|+\left|x_{n}^{k}(t)-x_{n}^{k}\left(\tau_{i}^{\epsilon}\left(x_{n}^{k}\right)\right)\right|+\left|x_{n}^{k}\left(\tau_{i}^{\epsilon}\left(x_{n}^{k}\right)\right)-x^{k}\left(r_{i}^{k}\right)\right| \\
& \leq \eta / 2+\epsilon+\eta / 2=\epsilon+\eta .
\end{aligned}
$$

Since $\eta$ was arbitrary, $\left|x^{k}(t)-x^{k}\left(r_{i}^{k}\right)\right| \leq \epsilon$. 
Finally since $\epsilon \leq\left|x_{n}^{k}\left(\tau_{i}^{\epsilon}\left(x_{n}^{k}\right)\right)-\tau_{i+1}^{\epsilon}\left(x_{n}^{k}\right)\right|$, taking limits as $n \rightarrow \infty$ and using the continuity of $x^{k}$, we obtain $\left|x\left(r_{i}^{k}\right)-x\left(r_{i+1}^{k}\right)\right|=\epsilon$.

Theorem 7.3 $\left\{\left(\left(X_{n}, Y_{n}\right), G_{\epsilon}\left(X_{n}, Y_{n}\right)\right)\right\}$ satisfies the large deviation principle with good rate function $I_{0}$ defined by:

$$
I_{0}(x, y, z)=\left\{\begin{array}{rll}
I^{\sharp}(x, y) & : & z \in G_{\epsilon}^{(x, y)}, \\
\infty & : & \text { otherwise }
\end{array}\right.
$$

In particular, $\left\{G_{\epsilon}\left(X_{n}, Y_{n}\right)\right\}$ satisfies the large deviation principle with good rate function I given by:

$$
I(z)=\inf \left\{I_{0}(x, y, z): z \in G_{\epsilon}^{(x, y)}\right\}=\inf \left\{I^{\sharp}(x, y): z \in G_{\epsilon}^{(x, y)}\right\} .
$$

Proof: Theorem 7.3 will hold if we can check the second condition of Theorem 1.1, for $G_{\epsilon}$ and $I^{\sharp}$.

Let $x, y \in H_{1}, z \in G_{\epsilon}^{(x, y)}$. Let $\left\{r_{i}^{k}\right\}$ be as in Lemma 7.2 and let $f$ be the derivative of $x$. Define the sequences $\delta_{i}^{n, k}, s_{i}^{n, k}, t_{i}^{n, k}, a_{i}^{n, k}, l_{i}^{n, k}, u_{i}^{n, k}, w_{i}^{n, k}, \Delta_{i}^{k} x, f_{n}^{k}, x_{n}^{k}$ as follows: $\delta_{i}^{n, k}, a_{i}^{n, k} \rightarrow 0$ as $n \rightarrow \infty, \quad \delta_{0}^{n, k}=0, \quad s_{i}^{n, k}=r_{i}^{k}+\delta_{i}^{n, k}, \quad t_{i}^{n, k}=r_{i}^{k}+2 \delta_{i}^{n, k}, \quad\left|x^{k}\left(t_{i}^{n, k}\right)-x^{k}\left(r_{i}^{k}\right)\right|<\epsilon / 2$, and $\Delta_{i}^{k} x=x^{k}\left(r_{i}^{k}\right)-x^{k}\left(r_{i-1}^{k}\right)$.

$u_{i}^{n, k}(t)$ is the line joining $\left(t_{i}^{n, k}, 1-1 / n\right)$ and $\left(r_{i+1}^{k}, 1\right)$ if $t \in\left[t_{i}^{n, k}, r_{i+1}^{k}\right]$ and 0 otherwise. $w_{i}^{n, k}$ is the slope of $u_{i}^{n, k}$.

$l_{i}^{n, k}(t)$ is the polygonal function joining the three points $\left(r_{i}^{k}, x^{k}\left(r_{i}^{k}\right)\right), \quad\left(s_{i}^{n, k}, x^{k}\left(r_{i}^{k}\right)+\right.$ $\left.a_{i}^{n, k} \Delta_{i}^{k} x\right)$ and $\left(t_{i}^{n, k}, x^{k}\left(r_{i}^{k}\right)+(1-1 / n)\left[x^{k}\left(t_{i}^{n, k}\right)-x^{k}\left(r_{i}^{k}\right)\right]\right)$ if $t \in\left[r_{i}^{k}, t_{i}^{n, k}\right]$ and 0 otherwise.

$$
\begin{aligned}
& f_{n}^{k}(t)=\left\{\begin{array}{ll}
\left(l_{i}^{n, k}\right)^{\prime}(t) & : \quad t \in\left[r_{i}^{k}, t_{i}^{n, k}\right), \\
u_{i}^{n, k}(t) f^{k}(t)+w_{i}^{n, k} \int_{r_{i}^{k}}^{t} f^{k}(s) d s & : \quad t \in\left[t_{i}^{n, k}, r_{i+1}^{k}\right)
\end{array} .\right. \\
& x_{n}^{k}(t)=\int_{0}^{t} f_{n}^{k}(s) d s, y_{n}^{k} \equiv y^{k} . \\
& x_{n}^{k}(t)=u_{0}^{n, k}(t) x^{k}(t) I_{\left[0, r_{1}^{k}\right)}(t)+l_{1}^{n, k}(t) I_{\left[r_{1}^{k}, t_{1}^{n, k}\right)}(t) \\
& +\left\{x^{k}\left(r_{1}^{k}\right)+u_{1}^{n, k}(t)\left[x^{k}(t)-x^{k}\left(r_{1}^{k}\right)\right]\right\} I_{\left[t_{1}^{n, k}, r_{2}^{k}\right)}(t)+l_{2}^{n, k}(t) I_{\left[r_{2}^{k}, t_{2}^{n, k}\right)}(t)+\ldots
\end{aligned}
$$


$x_{n}^{k}$ is an approximation to $x^{k}$ as in the following figures:

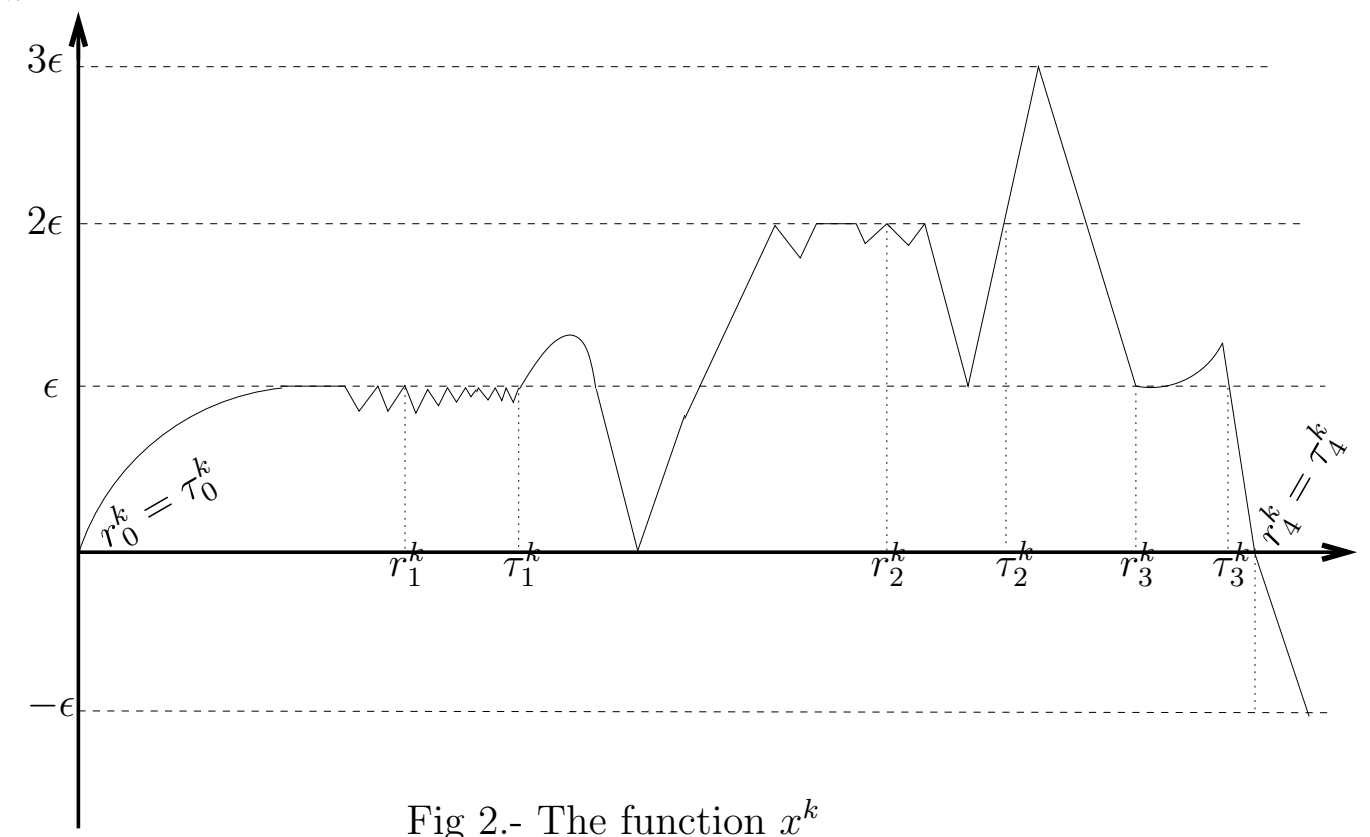

Fig 2.- The function $x^{k}$

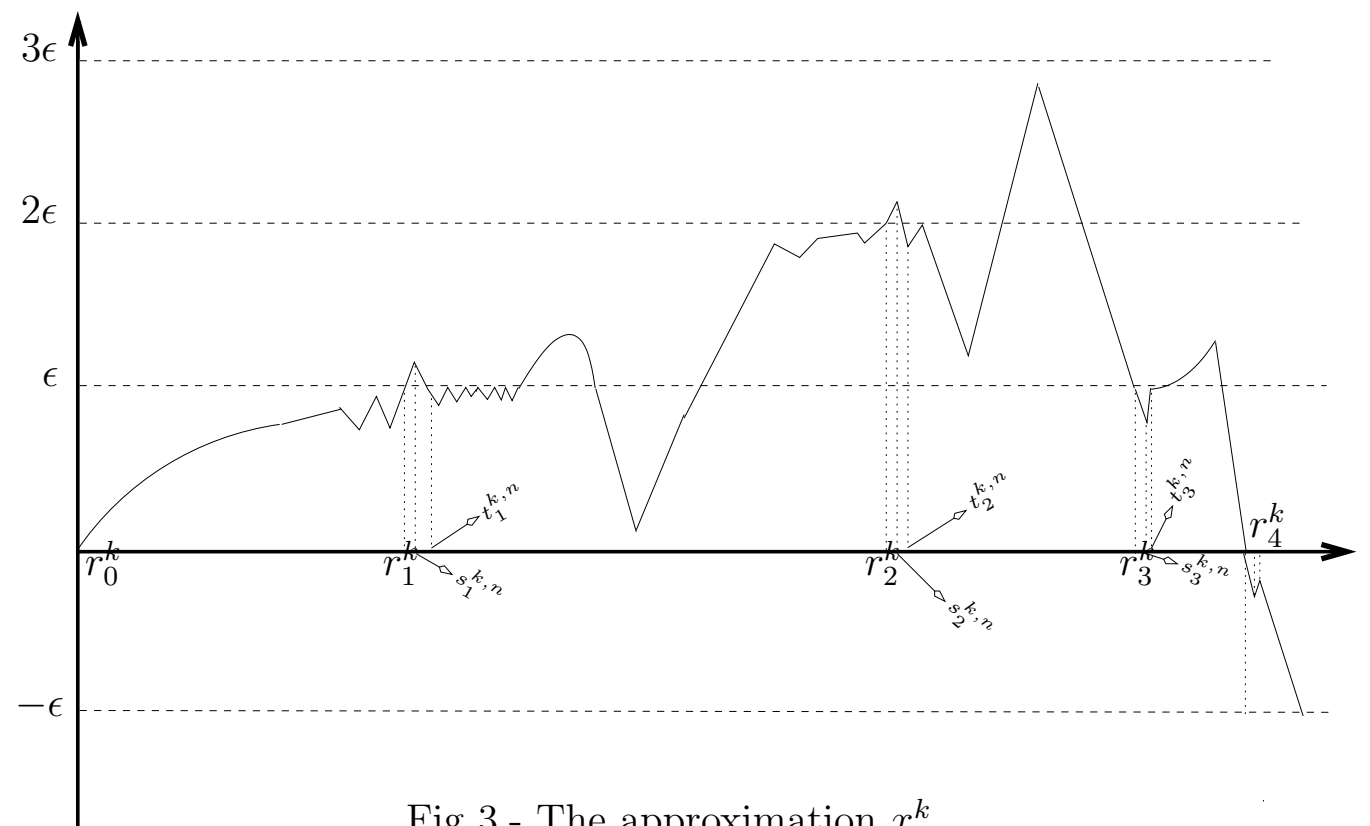

Fig 3.- The approximation $x_{n}^{k}$

We can see that if $t \in\left[0, r_{1}^{k}\right)$, then $\left|x_{n}^{k}(t)-x_{n}^{k}(0)\right|<\epsilon$, and if $t \in\left(r_{1}^{k}, s_{1}^{n, k}\right]$, then $\left|x_{n}^{k}(t)-x_{n}^{k}(0)\right|>\epsilon$. Hence $\tau_{1}^{\epsilon}\left(x_{n}^{k}\right)=r_{1}^{k}$. Similarly, for each $i, \tau_{i}^{\epsilon}\left(x_{n}^{k}\right)=r_{i}^{k}$ and for all $t \in\left[r_{i}^{k}, r_{i+1}^{k}\right),\left|x_{n}^{k}(t)-x_{n}^{k}\left(r_{i}^{k}\right)\right|<\epsilon$. We also have that $x_{n}^{k}\left(r_{i}^{k}\right)=x^{k}\left(r_{i}^{k}\right)$. 
Claim $G_{\epsilon}$ is continuous at $\left(x_{n}, y_{n}\right)$.

Proof (claim) Let $\hat{z}$ in $G_{\epsilon}^{\left(x_{n}, y_{n}\right)}$. We proceed as before. Using Lemma 7.2, for each $k \in\{1 \ldots d\}$, there is a sequence $\left\{q_{i}^{k}\right\}$ such that

1. $\left\{q_{i}^{k}\right\} \subseteq[0, \infty], q_{0}^{k}=0, q_{i}^{k} \uparrow \infty$

2. For all $t$ in $\left[q_{i}^{k}, q_{i+1}^{k}\right),\left|x_{n}^{k}(t)-x_{n}^{k}\left(q_{i}^{k}\right)\right| \leq \epsilon$

3. For all $i,\left|x_{n}^{k}\left(q_{i}^{k}\right)-x_{n}^{k}\left(q_{i+1}^{k}\right)\right|=\epsilon$,

and

$$
\hat{z}^{k}(t)=\sum_{i=1}^{j} x_{n}^{k}\left(q_{i-1}^{k}\right)\left(y_{n}^{k}\left(q_{i}^{k}\right)-y_{n}^{k}\left(q_{i-1}^{k}\right)\right)+x_{n}^{k}\left(q_{j}^{k}\right)\left(y_{n}^{k}(t)-y_{n}^{k}\left(q_{j}^{k}\right)\right), \quad t \in\left[q_{j}^{k}, q_{j+1}^{k}\right) .
$$

In particular the fact that for all $t$ in $\left[q_{i}^{k}, q_{i+1}^{k}\right),\left|x_{n}^{k}(t)-x_{n}^{k}\left(q_{i}^{k}\right)\right| \leq \epsilon$ implies that $q_{1}^{k} \leq$ $r_{1}^{k} \equiv \tau_{1}^{\epsilon}\left(x_{n}^{k}\right)$, and since $\left|x_{n}^{k}\left(q_{1}^{k}\right)-x_{n}^{k}\left(q_{0}\right)\right|=\left|x_{n}^{k}\left(q_{i}^{k}\right)-x_{n}^{k}(0)\right|=\epsilon$, then $r_{1}^{k} \leq q_{1}^{k}$ (otherwise taking $t=q_{1}^{k}<r_{1}^{k}$ would imply $\left|x_{n}^{k}(t)-x_{n}^{k}(0)\right|<\epsilon$ which is impossible). Therefore $q_{1}^{k}=r_{1}^{k}$, and similarly $q_{i}^{k}=r_{i}^{k}$. Therefore

$$
\hat{z}^{k}(t)=\sum_{i=1}^{j} x_{n}^{k}\left(r_{i-1}^{k}\right)\left(y_{n}^{k}\left(r_{i}^{k}\right)-y_{n}^{k}\left(r_{i-1}^{k}\right)\right)+x_{n}^{k}\left(r_{j}^{k}\right)\left(y_{n}^{k}(t)-y_{n}^{k}\left(r_{j}^{k}\right)\right), \quad t \in\left[r_{j}^{k}, r_{j+1}^{k}\right),
$$

i.e. $\hat{z}^{k}=g_{\epsilon}\left(x_{n}^{k}, y_{n}^{k}\right)$. Using Lemma 7.1 we conclude that $G_{\epsilon}$ is continuous at $\left(x_{n}, y_{n}\right)$, which proves our claim.

Let $t \in\left[r_{i}^{k}, t_{i}^{n, k}\right)$. Then

$$
\begin{aligned}
\left|x_{n}^{k}(t)-x^{k}(t)\right| & =\left|l_{i}^{n, k}(t)-x^{k}(t)\right| \\
& \leq\left|l_{i}^{n, k}(t)-x^{k}\left(r_{i}^{k}\right)\right|+\left|x^{k}\left(r_{i}^{k}\right)-x^{k}(t)\right| .
\end{aligned}
$$

Both quantities are small by the continuity of $x$ and the definition of $l_{i}^{n, k}$. Similarly, if $t \in\left[t_{i}^{n, k}, r_{i+1}^{k}\right)$, then

$$
\left|x_{n}^{k}(t)-x^{k}(t)\right|=\left|x^{k}\left(r_{i}^{k}\right)+u_{i}^{n, k}(t)\left[x^{k}(t)-x^{k}\left(r_{i}^{k}\right)\right]-x^{k}(t)\right|
$$




$$
\begin{aligned}
& =\left|x^{k}(t)-x^{k}\left(r_{i}^{k}\right)\right| \cdot\left|1-u_{i}^{n, k}(t)\right| \\
& \leq \frac{1}{n}\left|x^{k}(t)-x^{k}\left(r_{i}^{k}\right)\right|,
\end{aligned}
$$

which is also small. Therefore for $t \in\left[r_{i}^{k}, r_{i+1}^{k}\right)$ we can make $\left|x_{n}^{k}(t)-x^{k}(t)\right|$ small if $n$, is large enough.

Of course $\left(x_{n}, y_{n}\right) \rightarrow(x, y)$ uniformly on bounded time intervals.

Also $g_{\epsilon}\left(x_{n}^{k}, y_{n}^{k}\right) \rightarrow \sum_{i=1}^{\infty} x^{k}\left(r_{i-1}^{k}\right)\left(y^{k}\left(r_{i}^{k} \wedge t\right)-y^{k}\left(r_{i-1}^{k} \wedge t\right)\right)=g_{\epsilon}\left(x^{k}, y^{k}\right)$ uniformly on bounded time intervals. Therefore to check the second condition of Theorem (1.1), it is left to check that:

$$
I^{\sharp}\left(x_{n}, y_{n}\right)=\int_{0}^{\infty}\left(\left\|\dot{x_{n}}(t)\right\|^{2}+\left\|\dot{y_{n}}(t)\right\|^{2}\right) d t \rightarrow I^{\sharp}(x, y)
$$

Now

$$
\begin{aligned}
\int_{0}^{\infty}\left(\dot{x}_{n}^{k}(t)\right)^{2} d t=\int_{0}^{\infty}\left(f_{n}^{k}(t)\right)^{2} d t \\
\quad=\sum_{i=0}^{\infty}\left\{\int_{r_{i}^{k}}^{t_{i}^{n, k}}\left[\left(l_{i}^{n, k}\right)^{\prime}(t)\right]^{2} d t+\int_{t_{i}^{n, k}}^{r_{i+1}^{k}}\left[u_{i}^{n, k}(t) f^{k}(t)+w_{i}^{n, k} \int_{r_{i}^{k}}^{t} f^{k}(s) d s\right]^{2} d t\right\} \\
\quad=\sum_{i=0}^{\infty}\left\{\int_{r_{i}^{k}}^{s_{i}^{n, k}}\left[\left(l_{i}^{n, k}\right)^{\prime}(t)\right]^{2} d t+\int_{s_{i}^{n, k}}^{t_{i}^{n, k}}\left[\left(l_{i}^{n, k}\right)^{\prime}(t)\right]^{2} d t+\int_{r_{i}^{k}}^{r_{i+1}^{k}}\left[A_{i}^{n, k}(t)\right]^{2} d t\right\}
\end{aligned}
$$

where

$$
A_{i}^{n, k}(t)=u_{i}^{n, k}(t) f^{k}(t) I_{\left[t_{i}^{n, k}, r_{i+1}^{k}\right)}(t)+w_{i}^{n, k} \int_{r_{i}^{k}}^{t} f^{k}(s) d s \cdot I_{\left[t_{i}^{n, k}, r_{i+1}^{k}\right)}(t) .
$$

Since $u_{i}^{n, k}(t) \rightarrow 1, I_{\left[t_{i}^{n, k}, r_{i+1}^{k}\right)}(t) \rightarrow I_{\left[r_{i}^{k}, r_{i+1}^{k}\right)}(t)$ and $w_{i}^{n, k} \rightarrow 0$ as $n \rightarrow \infty, A_{i}^{n, k}(t) \rightarrow f^{k}(t)$ on $\left[r_{i}^{k}, r_{i+1}^{k}\right)$ as $n \rightarrow \infty$; by the dominated convergence theorem, if $n \rightarrow \infty$,

$$
\int_{r_{i}^{k}}^{r_{i+1}^{k}}\left[A_{i}^{n, k}(t)\right]^{2} d t \rightarrow \int_{r_{i}^{k}}^{r_{i+1}^{k}}\left(f^{k}(t)\right)^{2} d t .
$$

We will prove that the other terms in (7.4) go to 0 as $n \rightarrow \infty$ :

$$
\begin{aligned}
\sum_{i=0}^{\infty} \int_{r_{i}^{k}}^{s_{i}^{n, k}}\left[\left(l_{i}^{n, k}\right)^{\prime}(t)\right]^{2} d t & =\sum_{i=0}^{\infty} \int_{r_{i}^{k}}^{s_{i}^{n, k}} \frac{\left(a_{i}^{n, k}\right)^{2}\left(\Delta_{i}^{k} x\right)^{2}}{\left(\delta_{i}^{n, k}\right)^{2}} d t \\
& =\sum_{i=0}^{\infty} \frac{\left(a_{i}^{n, k}\right)^{2} \epsilon^{2}}{\delta_{i}^{n, k}} .
\end{aligned}
$$


If $a_{i}^{n, k}$ satisfies $\left(a_{i}^{n, k}\right)^{2}=\frac{\delta_{i}^{n, k}}{n i^{2}}$, then the last term in the previous equation will be a finite number that goes to 0 as $n \rightarrow \infty$. For the other term, we proceed carefully:

$$
\begin{gathered}
\sum_{i=0}^{\infty} \int_{s_{i}^{n, k}}^{t_{i}^{n, k}}\left[\left(l_{i}^{n, k}\right){ }^{\prime}(t)\right]^{2} d t=\sum_{i=0}^{\infty} \int_{s_{i}^{n, k}}^{t_{i}^{n, k}}\left[\frac{(1-1 / n)\left[x^{k}\left(t_{i}^{n, k}\right)-x^{k}\left(r_{i}^{k}\right)\right]-a_{i}^{n, k} \Delta_{i}^{k} x}{\delta_{i}^{n, k}}\right]^{2} d t \\
\leq \sum_{i=0}^{\infty} \int_{s_{i}^{n, k}}^{t_{i}^{n, k}}\left\{2\left[\frac{(1-1 / n)\left[x^{k}\left(t_{i}^{n, k}\right)-x^{k}\left(r_{i}^{k}\right)\right]}{\delta_{i}^{n, k}}\right]^{2}+2\left[\frac{a_{i}^{n, k} \Delta_{i}^{k} x}{\delta_{i}^{n, k}}\right]^{2}\right\} d t \\
\leq \sum_{i=0}^{\infty} \int_{s_{i}^{n, k}}^{t_{i}^{n, k}} 2\left[\frac{(1-1 / n)\left[x^{k}\left(t_{i}^{n, k}\right)-x^{k}\left(r_{i}^{k}\right)\right]}{\delta_{i}^{n, k}}\right]^{2} d t+2 \sum_{i=0}^{\infty} \frac{\left(a_{i}^{n, k}\right)^{2} \epsilon^{2}}{\delta_{i}^{n, k}} .
\end{gathered}
$$

Consider now an application of Jensen's inequality in the first of our above terms:

$$
\begin{aligned}
\int_{s_{i}^{n, k}}^{t_{i}^{n, k}} 2\left[\frac{(1-1 / n)\left[x^{k}\left(t_{i}^{n, k}\right)-x^{k}\left(r_{i}^{k}\right)\right]}{\left.\delta_{i}^{n, k}\right]^{2} d t}\right. & \leq 2 \delta_{i}^{n, k} \cdot\left[\frac{x^{k}\left(t_{i}^{n, k}\right)-x^{k}\left(r_{i}^{k}\right)}{\delta_{i}^{n, k}}\right]^{2} \\
& =2 \delta_{i}^{n, k} \cdot\left[\frac{1}{\delta_{i}^{n, k}} \int_{r_{i}^{k}}^{t_{i}^{n, k}} f^{k}(s) d s\right]^{2} \\
& \leq 2 \delta_{i}^{n, k} \cdot \frac{1}{\delta_{i}^{n, k}} \int_{r_{i}^{k}}^{t_{i}^{n, k}}\left(f^{k}(s)\right)^{2} d s \\
& =2 \int_{r_{i}^{k}}^{t_{i}^{n, k}}\left(f^{k}(s)\right)^{2} d s
\end{aligned}
$$

The previous argument implies that

$$
\lim _{n \rightarrow \infty} \sum_{i=0}^{\infty} \int_{s_{i}^{n, k}}^{t_{i}^{n, k}} 2\left[\frac{(1-1 / n)\left[x^{k}\left(t_{i}^{n, k}\right)-x^{k}\left(r_{i}^{k}\right)\right]}{\delta_{i}^{n, k}}\right]^{2} d t=0 .
$$

Hence

$$
\int_{0}^{\infty}\left(\dot{x_{n}}{ }^{k}(t)\right)^{2} d t \stackrel{n \rightarrow \infty}{\longrightarrow} \sum_{i=0}^{\infty} \int_{r_{i}^{k}}^{r_{i+1}^{k}}\left(f^{k}(t)^{2} d t=\int_{0}^{\infty}\left(\dot{x}^{k}(t)\right)^{2} d t\right.
$$

and consequently the second condition of Theorem 1.1 is fully checked. 


\section{References}

[1] M. A. Arcones, The large deviation principle of stochastic processes. preprint, 2000.

[2] A. Dembo and O. Zeitouni, Large Deviations Techniques And Applications, Springer Verlag, New York, second edition ed., 1998.

[3] J. Deuschel And D. Stroock, Large Deviations, American Mathematical Society, Providence, Rhode Island, 2000.

[4] M. Gangster And J. Dontchev, More on mild continuity, Rend. Insti. Mat. Univ. Trieste, 27 (1995), pp. 47-59.

[5] J. GarciA, A large deviations principle for stochastic integrals. Preprint, 2003.

[6] J. L. Kelley, General Topology, D. Van Nostrand Company, Canada, 1955.

[7] S. Kempisty, Sur les fonctions quasicontinues, Fund. Math., 19 (1932), pp. 184-197.

[8] P.Eichelsbacher And U. Schmock, Exponential approximations in completely regular topological spaces and extensions of sanovs theorem, Stoch. Processes and its Applications, 77 (1998), pp. 233-251.

[9] A. Puhalksis, On functional principle of large deviations, New trends in probability and statistics, 1 (1991), pp. 198-218.

[10] _ Large deviation analysis of the single server queue, Queueing Systems, 21 (1995), pp. $5-66$.

[11] S. R. S. Varadhan, Asymptotic probabilities and differential equations, Comm. Pure Appl. Math., 19 (1966), pp. 261-286. 